\title{
Structure of Some von Neumann Algebras with Isolated Discrete Modular Spectrum
}

By

\author{
Huzihiro Araki
}

\begin{abstract}
From a finite von Neumann algebra $\mathfrak{F}$ with a faithful normal trace and its normal injective $*$ endomorphism $\phi$ satisfying $\phi(\Re)=\phi(1) \Im \phi(1)$, we construct another von Neumann algebra $M(\mathfrak{F}, \phi)$ by a method which reduces to groupmeasure construction when $\mathfrak{F}$ is commutative and $\phi$ is an automorphism. If $\phi$ satisfies $\phi(z)=\phi(1) z$ for all central elements $z$ of $\mathfrak{F}$ and $\phi(1)^{\natural}=e^{-a}$ for a positive number $a$, then $M(\mathfrak{F}, \phi)$ has the following 3 properties: (1) It has a faithful normal state $\rho$ whose modular operator $\Delta_{\rho}$ has the spectrum $\{0\} \cup\left\{e^{n a}\right.$; $n=0, \pm 1, \ldots\}=S_{e}$. (2) The set $\mathfrak{M}_{0}$ of all elements of $M(\mathfrak{F}, \phi)$, commuting with $\Delta_{\rho}$ is isomorphic to $\mathfrak{F}$. (3) The center of $\mathfrak{M}_{0}$ coincides with the center of $\mathfrak{M}$.

Conversely, any von Neumann algebra with a faithful normal state $\rho$ such that $\log \Delta_{\rho}$ has exclusively an isolated point spectrum and the center of $\mathfrak{M}_{0}$ coincides with its center is a direct sum of $M\left(\mathfrak{F}_{j}, \phi_{j}\right), j=1, \ldots$, and possibly a finite von Neumann algebra, where each $\phi_{j}$ satisfies $\phi_{j}(\mathfrak{F})=\phi_{j}(1) \mathfrak{F} \phi_{j}(1)$, $\phi_{j}(z)=z \phi_{j}(1)$ for all central element $z$ of $\mathfrak{F}$ and $\phi_{j}(1)^{\natural}=e^{-a_{j}}$.

If $\rho$ is a $K M S$ state under time translation of a $C^{*}$ algebra, which is asymptotically abelian with respect to (either discrete or continuous) space translation and if the spectrum of generator of time translation has exclusively an isolated point spectrum in the representation associated with $\rho$, then the associated von Neumann algebra has the above structure where the asymptotic ratio set of $\mathfrak{F}_{j}$ as well as that of a possible finite summand (if non-zero) is $\{1\}$ and $r_{\infty}\left(M\left(\mathfrak{F}_{j}, \phi_{j}\right)\right)=S_{x_{j}}$ where $x_{j}=e^{a_{j}}$. The last result on asymptotic ratio set is limited to the case where the representation space is separable.

A generalization of $M(\mathfrak{F}, \phi)$ for a commutative semigroup of endomorphisms of a finite von Neumann algebra, instead of one $\phi$, is given.
\end{abstract}

\section{§1. Notation and Main Results}

Let $\mathfrak{M}$ be a von Neumann algebra, $\Omega$ be a cyclic and separating unit

* Received August 4, 1972. 
vector, $\Delta$ be the modular operator for $\Omega, \tau(t) Q=\Delta^{i t} Q \Delta^{-i t}, J$ be the modular conjugation operator for $\Omega$ and $j(Q)=J Q J$.

$\mathfrak{S}_{\alpha}$ denotes the eigenspace of $\log \Delta$ belonging to an eigenvalue $\alpha$ and $\mathfrak{M}_{\alpha}$ denotes the set of $Q \in \mathfrak{M}$ such that $\tau(t) Q=e^{i t \alpha} Q$. It is known that $\mathfrak{M}_{0}$ is a finite von Neumann algebra containing the center 3 of $\mathfrak{M}$ and $\Omega$ is a cyclic and separating trace vector for $\mathfrak{M}_{0}$ restricted to $\mathfrak{L}_{0} . \mathfrak{Z}_{0}$ denotes the center of $\mathfrak{M}_{0} . Z_{0} \supset 3$.

We are interested in the structure of $\mathfrak{M}$ and we can analyze it when $\log \Delta$ has exclusively an isolated point spectrum and $3_{0}=3$.

For any given finite von Neumann algebra $\mathfrak{F}$ with a faithful normal trace vector $\Psi$ such as $\mathfrak{M}_{0}$ with $\Omega$ and its normal injective * endomorphism $\phi$ satisfying $\phi(\mathfrak{F})=\phi(1) \Im \phi(1)$, we present in section 2 a method of constructing another von Neumann algebra, denoted as $M(\mathfrak{F}, \phi)$, with a cyclic and separating vector $\Omega(\mathfrak{F}, \phi)$. If $(\Psi, \phi(z) \Psi)=e^{-a}(\Psi, z \Psi)$ for all $z \in \mathfrak{F}_{c}$ (the center of $\mathfrak{F}$ ), then the spectrum of modular operator $\Delta(\mathfrak{F}, \phi)$ for $\Omega(\mathfrak{F}, \phi)$ is

$$
S_{e^{a}}=\{0\} \cup\left\{e^{n a} ; n=0, \pm 1, \ldots\right\}
$$

If $\phi(z)=z \phi(1)$ for $z \in \mathfrak{F}_{c}$, then $\mathfrak{Z}_{0}=\mathbb{Z}$.

The following main result proved in section 3 gives a converse.

Theorem 1. Let $\mathfrak{M}$ be a von Neumann algebra with a cyclic and separating vector $\Omega$ such that $\log \Delta$ has exclusively an isolated point spectrum

$$
\left\{0, \pm a_{1}, \pm a_{2}, \ldots\right\}, 0<a_{1}<a_{2} \ldots
$$

and the center $\mathfrak{Z}_{0}$ of the set $\mathfrak{M}_{0}$ of all elements in $\mathfrak{M}$ commuting with $\Delta$ coincides with the center 3 of $\mathfrak{M}$. Then there exists central projections $P_{n}$ of $\mathfrak{M}, n=0,1, \ldots$ such that

(i) $P_{n} \perp P_{m}$ for $n \neq m, \Sigma P_{n}=1$ and $P_{1} \neq 0$,

(ii) $P_{0} \mathfrak{M}$ is finite if $P_{0} \neq 0$,

(iii) $P_{n} \mathfrak{M}$ is * isomorphic to $M\left(P_{n} \mathfrak{M}_{0}, \phi_{n}\right)$ for some normal injective * endomorphism $\phi_{n}$ of $P_{n} \mathfrak{M}_{0}$ satisfying

$$
\phi_{n}\left(P_{n} \mathfrak{M}_{0}\right)=\phi_{n}\left(P_{n}\right) P_{n} \mathfrak{M}_{0} \phi_{n}\left(P_{n}\right)
$$




$$
\begin{aligned}
& \phi_{n}(z)=\phi_{n}(1) z, z \in P_{n} 3, \\
& \phi_{n}\left(P_{n}\right)^{\natural}=e^{-a_{n}} P_{n}
\end{aligned}
$$

if $P_{n} \neq 0$, where $h^{\text {is }}$ the canonical mapping in $\mathfrak{M}_{0}$.

In a special case where $\mathfrak{M}$ is a factor, the spectrum of $\log \Delta$ is necessarily an additive group. (This statement is always true if all subspaces $\mathfrak{S}(I)=\left(E_{\beta-0}-E_{\alpha+0}\right) \mathfrak{S}$ for $\Delta=\int \lambda d E_{\lambda}, I=(\alpha, \beta)$, is cyclic for $\mathfrak{M}$ (and hence separating for $\mathfrak{M}$ due to $J \mathfrak{S}(I)=\mathfrak{S}(-I)$ ) even when $\Delta$ has a continuous spectrum.)

Examples where the center of $\mathfrak{M}_{0}$ does not coincide with the center of $\mathfrak{M}$ are tensor product of ITPFI of the class $S_{01}$ (type III or II) with any finite von Neumann algebra where the vector $\Omega$ is product of a defining product vector of the ITPFI with a cyclic and separating trace vector for the finite von Neumann algebra. Another example for $3_{0} \neq 3$ is $R_{x} \otimes R$ with $\Omega=\Omega_{x} \otimes \Phi$ where $\Omega_{x}$ is the defining product vector of $R_{x}, R$ is type $I_{2}$ and spectrum of modular operator for $(R, \Phi)$ is $\left\{y^{-1}, 1, y\right\}$ with $y \notin S_{x}$.

The condition $3_{0}=3$ is satisfied for a KMS state of an asymptotically abelian $C^{*}$ algebra. More precisely, a net of operator $Q_{\alpha}$ in a von Neumann algebra $\mathfrak{M}$ is called strongly central if there exists a weakly total self-adjoint subset $\mathfrak{W}$ of $\mathfrak{M}$ such that $\lim _{\alpha}\left[Q_{\alpha}, w\right]=0$ strongly for every $w \in \mathfrak{W}$. A subset $\mathfrak{U}$ of $\mathfrak{M}$ is called strongly $\tau_{\alpha}$ central relative to a net $\tau_{\alpha}$ of $*$ automorphisms of $\mathfrak{M}$ if $\tau_{\alpha} Q$ is strongly central in $\mathfrak{M}$ for each $Q \in \mathfrak{Q}$. We have

Theorem 2. Let $\mathfrak{M}$ be a von Neumann algebra, $\tau_{\alpha}$ be a net of * automorphisms of $\mathfrak{M}, \rho$ be a faithful normal $\tau_{\alpha}$ invariant state of $\mathfrak{M}$ and श् be a weakly dense $C^{*}$ subalgebra of $\mathfrak{M}$, which is invariant under modular automorphism $\tau_{\rho}(t)$ for $\rho$ and is strongly $\tau_{\alpha}$ central. Assume that modular operator $\Delta_{\rho}$ for $\rho$ is such that $\log \Delta_{\rho}$ has exclusively an isolated point spectrum.

Then $3_{0}=3$ and there exists central projections $P_{n}$ satisfying (i) (iii) of Theorem 1. If the representation space is separable, in addition, then

$$
\begin{array}{lll}
r_{\infty}\left(P_{0} \mathfrak{M}\right)=\{1\} \quad \text { if } & P_{0} \neq 0, \\
r_{\infty}\left(P_{n} \mathfrak{M}_{0}\right)=\{1\} & \text { if } \quad & P_{n} \neq 0,
\end{array}
$$




$$
r_{\infty}\left(P_{n} \mathfrak{M}\right)=S_{x_{n}} \quad \text { if } \quad P_{n} \neq 0 \text {, where } x_{n}=e^{-a_{n}}, n>0 .
$$

This theorem is applicable to a situation where $\rho$ is a $K M S$ state for time translation of a $C^{*}$ algebra $A$, which is asymptotically abelian for (discrete or continuous) space translation, the generator of time translation has exclusively an isolated point spectrum in the representation associated with $\rho$ and the representation space is separable.

\section{§2. Construction of $M(\mathfrak{\mho}, \phi)$}

Let $\mathfrak{F}$ be a finite von Neumann algebra acting on a Hilbert space $\Re$ with a cyclic and separating unit trace vector $\Psi$ and $\widetilde{F}_{c}$ be the center of $\mathfrak{F}$. Let $\sharp$ be the canonical $\sharp$ mapping on $\mathfrak{\mho}_{c}, J_{T}$ be the modular conjugation operator for $\Psi$ and $j_{\Psi}(Q)=J_{\Psi} Q J_{\Psi}$.

Let $\phi$ be a normal injective $*$ endomorphism of $\widetilde{F}, \omega_{\Psi}$ be the vector state by $\Psi$, and $\phi^{*} \omega_{\Psi}$ be the normal positive linear functional defined by

$$
\phi^{*} \omega_{\Psi}(Q)=\omega_{\Psi}(\phi(Q))
$$

Both $\omega_{\Psi}$ and $\phi^{*} \omega_{\Psi}$ are faithful and tracial.

By the Radon-Nikodym theorem, there exists a strictly positive selfadjoint operator $A_{\phi}=\int \lambda d E_{\lambda}^{A}$ such that $E_{\lambda}^{A} \in \mathfrak{F}_{c}$ and

$$
\lim _{L \rightarrow \infty} \phi^{*} \omega_{\Psi}\left(A_{L} z A_{L}\right)=\omega_{\Psi}(z), A_{L}=A_{\phi} E_{L}^{A}, z \in \mathfrak{\mho}_{c} .
$$

In other words, $\Psi$ is in the domain of $\phi\left(A_{\phi}\right) \equiv \lim _{L} \phi\left(A_{L}\right)$ and

$$
\left(\phi\left(A_{\phi}\right) \Psi, \phi(z) \phi\left(A_{\phi}\right) \Psi\right)=(\Psi, z \Psi), z \in \mathfrak{F}_{c}
$$

(These equations hold for $z \in \mathfrak{F}$ as will be seen in the following proof.)

Lemma 1. There exists a unique isometric operator $V$ satisfying

$$
V Q \Psi=\phi(Q) \phi\left(A_{\phi}\right) \Psi . \quad Q \in \mathfrak{F}
$$

It satisfies

$$
\begin{aligned}
& V^{*} V=1, \\
& V Q=\phi(Q) V, \quad V^{*} \phi(Q)=Q V^{*}, \quad Q \in \mathfrak{F},
\end{aligned}
$$




$$
\left[J_{\Psi}, V\right]=0 \text {. }
$$

Proof. Because $\phi^{*} \omega_{\Psi}$ and $\omega_{\Psi}$ are tracial, we have

$$
\begin{aligned}
& \left\|\phi(Q) \phi\left(A_{L}\right) \Psi\right\|^{2}=\phi^{*} \omega_{\Psi}\left(A_{L} Q^{*} Q A_{L}\right) \\
& =\phi^{*} \omega_{\Psi}\left(\left(A_{L} Q^{*} Q A_{L}\right)^{\natural}\right) \\
& =\phi^{*} \omega_{\Psi}\left(A_{L}\left(Q^{*} Q\right)^{\natural} A_{L}\right) \\
& \rightarrow \omega_{\Psi}\left(\left(Q^{*} Q\right)^{\natural}\right)=\omega_{\Psi}\left(Q^{*} Q\right)=\|Q \Psi\|^{2}, \quad Q \in \mathfrak{F} .
\end{aligned}
$$

Since $\mathfrak{F} \Psi$ is dense in $\Re$, there exists a unique isometric $V$ satisfying (2.2). (2.3) says that $V$ is isometric.

The range of $V$ is the closure of $\phi(\mathfrak{F}) \phi\left(A_{\phi}\right) \Psi$, which is invariant under $\phi(\mathfrak{F})$. Hence $V V^{*}$ commutes with $\phi(Q), Q \in \mathfrak{F}$. From (2.2), we have for $Q \in \mathfrak{F}$ and $Q_{1} \in \mathfrak{F}$

$$
\begin{aligned}
V^{*} \phi(Q) V Q_{1} \Psi & =V^{*} \phi\left(Q Q_{1}\right) \phi\left(A_{\phi}\right) \Psi \\
& =V^{*} V Q Q_{1} \Psi=Q Q_{1} \Psi .
\end{aligned}
$$

Hence

$$
V^{*} \phi(Q) V=Q
$$

Hence

$$
\begin{aligned}
& V Q=V V^{*} \phi(Q) V=\phi(Q) V V^{*} V=\phi(Q) V, \\
& Q V^{*}=V^{*} \phi(Q) V V^{*}=V^{*} V V^{*} \phi(Q)=V^{*} \phi(Q) .
\end{aligned}
$$

Since $J_{\Psi} Q \Psi=Q * \Psi$, we have

$$
\begin{aligned}
V J_{\Psi} Q \Psi & =V Q * \Psi=\phi\left(Q^{*}\right) \phi\left(A_{\phi}\right) \Psi \\
& =\lim _{L \rightarrow \infty} \phi\left(Q A_{L}\right)^{*} \Psi=J_{\Psi} \phi(Q) \phi\left(A_{\phi}\right) \Psi \\
& =J_{\Psi} V Q \Psi
\end{aligned}
$$

Hence (2.5) holds.

Q.E.D. 
Lemma 2. If $z$ is a closed operator affiliated with $\mathfrak{F}$ and $\Psi$ is in the domain of $z$, then $\Psi$ is in the domain of $\phi\left(z A_{\phi}\right)$ and $V z \Psi=\phi\left(z A_{\phi}\right) \Psi$.

Proof. By polar decomposition of $z$, it is enough to prove the statement for a positive selfadjoint $z=\int \lambda d E_{\lambda}^{z}$.

Let $z_{\lambda}=z E_{\lambda}^{z}$. Then

$$
V z \Psi=\lim _{\lambda \rightarrow \infty} V z_{\lambda} \Psi=\lim _{\lambda \rightarrow \infty} \lim _{L \rightarrow \infty} \phi\left(z_{\lambda} A_{L}\right) \Psi
$$

Hence $\Psi$ is in the domain of $\phi\left(z A_{\phi}\right)$ and $V z \Psi=\phi\left(z A_{\phi}\right) \Psi$. (Note that $A_{\phi}$ is affiliated with $\mathfrak{F}_{c}$ and hence $E_{\lambda}^{z}$ and $E_{L}^{A}$ commute.) Q.E.D.

Lemma 3. Define

$$
\begin{aligned}
& D_{\phi}^{(n)}=\phi\left(D_{\phi}^{(n-1)} A_{\phi}\right), n=1,2, \ldots, \\
& D_{\phi}^{(0)}=1
\end{aligned}
$$

Then $V^{n} \Psi=D_{\phi}^{(n)} \Psi, D_{\phi}^{(n)}$ is affiliated with $\widetilde{\mho}$, commutes with $\phi^{n}(Q), Q \in \mathfrak{F}$ and its support $s\left(D_{\phi}^{(n)}\right)$ is $\phi^{n}(1)$.

Proof. By repeated use of Lemma 2, we have $V^{n} \Psi=D_{\phi}^{(n)} \Psi$. It is affiliated with $\widetilde{F}$ because it is a product of mutually commuting positive selfadjoint operators

$$
D_{\phi}^{(n)}=\prod_{k=1}^{n} \phi^{k}\left(A_{\phi}\right)
$$

Since spectral projections of $A_{\phi}$ is in the center of $\mathfrak{F}$, spectral projections of $\phi^{k}\left(A_{\phi}\right)$ is in the center of $\phi^{k}(\mathfrak{F}) \supset \phi^{n}(\mathfrak{F})(k \leqq n)$ and hence $D_{\phi}^{(n)}$ commutes with $\phi^{n}(Q), Q \in \mathfrak{F}$. Since $A_{\phi}$ is strictly positive, $s\left(A_{\phi}\right)=1$. If $z=$ $\int \lambda d E_{\lambda}$ is a positive selfadjoint operator, then $z \geqq \lambda\left(1-E_{\lambda}\right)$ implies $s(\phi(z))$ $\geqq \sup _{\lambda} \phi\left(1-E_{\lambda}\right)=\phi(s(z))$. Hence $s(\phi(z))=\phi(s(z))$. In particular,

$$
s\left(D_{\phi}^{(n)}\right)=\phi\left(s\left\{D_{\phi}^{(n-1)}\right\}\right)=\phi^{n}(1)
$$

by induction.

Q.E.D.

Lemma 4. Assume

$$
\phi(\mathfrak{F})=\phi(1) \mathfrak{F} \phi(1)
$$


Then $D_{\phi}^{(n)} \eta \phi^{n}(1) \mathfrak{F}_{c}$ and

$$
V^{n} V^{* n}=\phi^{n}(1) j_{\Psi}\left\{\phi^{n}(1)\right\}
$$

where $\eta$ denotes an operator affiliated with a von Neumann algebra.

Proof. From (2.7), $\phi^{k}\left(A_{\phi}\right) \eta \phi^{k}\left(\mathfrak{F}_{c}\right)=\mathfrak{F}_{c} \phi^{k}(1)$ and $\prod_{k=1}^{n} \phi^{k}(1)=\phi^{n}(1)$, we have $D_{\phi}^{(n)} \eta \phi^{n}(1) \mathfrak{F}_{c}$. The range of $V$ is the closure of

$$
\begin{aligned}
\phi(\mathfrak{F}) \Psi & =\phi(1) \mathfrak{F} \phi(1) \Psi=\phi(1) \mathfrak{F} J_{\Psi} \phi(1) \Psi \\
& =\phi(1) j_{\Psi}\{\phi(1)\} \mathfrak{F} \Psi
\end{aligned}
$$

and hence $V V^{*}=\phi(1) j_{\Psi}\{\phi(1)\}$.

By (2.4) and (2.5), we have inductively

$$
\begin{aligned}
V^{n} V^{* n} & =V \phi^{n-1}(1) j_{\Psi}\left\{\phi^{n-1}(1)\right\} V^{*} \\
& =\phi^{n}(1) j_{\Psi}\left\{\phi^{n}(1)\right\} V V^{*} \\
& =\phi^{n}(1) j_{\Psi}\left\{\phi^{n}(1)\right\} .
\end{aligned}
$$

Q.E.D.

We define

$$
\phi^{\prime}(y)=j_{\Psi}\left\{\phi\left(j_{\Psi}(y)\right)\right\}, y \in \mathfrak{F}^{\prime}
$$

Then from Lemma 1,

$$
V^{*} \phi^{\prime}(y)=y V^{*}, V y=\phi^{\prime}(y) V, y \in \widetilde{F}^{\prime}
$$

We also note

$$
\phi^{\prime n}(1)=j_{\Psi}\left\{\phi^{n}(1)\right\}
$$

We now construct $M(\mathfrak{F}, \phi)$ on a Hilbert space

$$
\mathfrak{Q}=\left\{\bigoplus_{-\infty}^{-1} \phi^{\prime n \mid}(1) \Re\right\} \oplus\left\{\oplus_{0}^{\infty} \phi^{n}(1) \Re\right\},
$$

namely we have partially isometric mappings $p_{n}$ from $\Re$ into $\mathfrak{S}$ such that

$$
p_{n}^{*} p_{n}= \begin{cases}\phi^{n}(1), & n \geqq 0, \\ \phi^{\prime|n|}(1), & n \leqq 0,\end{cases}
$$


and subspaces $\mathfrak{C}_{n} \equiv p_{n} \Re$ are mutually orthogonal and span the whole space S.

Let $x \in \mathfrak{F}, y \in \mathfrak{F}^{\prime}$. We define faithful representations of $\mathfrak{F}$ and $\mathfrak{F}^{\prime}$ by

$$
\begin{aligned}
& \pi(x)=\sum_{n} p_{n} \pi_{n}(x) p_{n}^{*}, \\
& \pi_{n}(x)= \begin{cases}\phi^{n}(x), & n \geqq 0, \\
x, & n \leqq 0,\end{cases} \\
& \pi^{\prime}(y)=\sum_{n} p_{n} \pi_{n}^{\prime}(y) p_{n}^{*}, \\
& \pi_{n}^{\prime}(y)= \begin{cases}y, & n \geqq 0 \\
\phi^{\prime|n|}(y), & n \leqq 0 .\end{cases}
\end{aligned}
$$

We also define partially isometric operators

$$
\begin{array}{r}
U=\sum_{n=-\infty}^{\infty} p_{n+1} U_{n} p_{n}^{*}, \\
U_{n}= \begin{cases}1, & n \geqq 0, \\
V^{*}, & n<0,\end{cases} \\
U^{\prime}=\sum_{n=-\infty}^{\infty} p_{n-1} U_{n}^{\prime} p_{n}^{*}, \\
U_{n}^{\prime}= \begin{cases}V^{*} & n>0, \\
1 & n \leqq 0 .\end{cases}
\end{array}
$$

The von Neumann algebra $M(\mathfrak{F}, \phi)$ and a candidate for its commutant are defined by

$$
\begin{aligned}
& M(\mathfrak{F}, \phi)=\left\{\pi(\mathfrak{F}), U, U^{*}\right\}^{\prime \prime}, \\
& M^{\prime}(\mathfrak{F}, \phi)=\left\{\pi^{\prime}\left(\mathfrak{F}^{\prime}\right), U^{\prime}, U^{\prime *}\right\}^{\prime \prime} .
\end{aligned}
$$

Theorem 3. Assume $\phi(F)=\phi(1) \mathfrak{F} \phi(1) . \quad \Omega(\mathfrak{F}, \phi) \equiv p_{0} \Psi$ is a cyclic and separating vector for $M(\mathfrak{F}, \phi)$, with modular operator $\Delta(\mathfrak{F}, \phi)$ and modular conjugation operator $J(\mathfrak{F}, \phi)$ given by

$$
\Delta(\mathfrak{F}, \phi)=\sum_{-\infty}^{-1} p_{n} j_{\Psi}\left\{D_{\phi}^{(|n|)}\right\}^{-2} p_{n}^{*}+\sum_{0}^{\infty} p_{n}\left\{D_{\phi}^{(n)}\right\}^{2} p_{n}^{*},
$$




$$
J(\mathfrak{F}, \phi)=\sum_{n} p_{-n} J_{\Psi} p_{n}^{*},
$$

where the inverse in $j_{\Psi}\left\{D^{(n)}\right\}^{-2}$ is to be taken in $\phi^{\prime n}(1) \Re=J_{\Psi} \phi^{n}(1) \Re$. $M(\mathfrak{F}, \phi)$ satisfies

$$
M(\mathfrak{\mho}, \phi)^{\prime}=M^{\prime}(\mathfrak{\mho}, \phi)
$$

If $A_{\phi}>1$, then the set of $Q \in M(\mathfrak{F}, \phi)$ commuting with $\Delta(\mathfrak{F}, \phi)$ is $\pi(\mathfrak{F})$. If $A_{\phi}=e^{a / 2}$ for a strictly positive number $a$, then the spectrum of $\Delta(\mathfrak{F}, \phi)$ is

$$
S_{x}=\left\{x^{n} ; n=0, \pm 1, \ldots\right\} \cup\{0\}, x=e^{a} .
$$

If $A_{\phi}>1$ and $\phi(z)=z \phi(1)$ for all $z \in \mathfrak{F}_{c}$, then the center of $\pi(\mathfrak{F})$ coincides with the center of $M(\mathfrak{F}, \phi)$. Under the assumption $A_{\phi}>1$, the center of $M(\mathfrak{F}, \phi)$ consists of all $z \in \mathfrak{F}_{c}$ such that $\phi(z)=z \phi(1)$,

If $\phi(z)=z \phi(1)$ for all $z \in \mathfrak{F}_{c}, A_{\phi}=\left\{\phi(1)^{\natural}\right\}^{-1 / 2}$.

Proof. $J(\mathfrak{F}, \phi)$ defined by $(2.25)$ is antiunitary and satisfies

$$
J(\mathfrak{F}, \phi)^{2}=1, J(\mathfrak{F}, \phi) \Omega(\mathfrak{F}, \phi)=\Omega(\mathfrak{F}, \phi),
$$$$
J(\mathfrak{F}, \phi) \pi(x) J(\mathfrak{F}, \phi)=\pi^{\prime}\left(j_{\Psi}(x)\right), x \in \mathfrak{F}
$$$$
J(\mathfrak{F}, \phi) U J(\mathfrak{F}, \phi)=U^{\prime},
$$

where (2.5) has been used in (2.30).

We obtain

$$
M^{\prime}(\mathfrak{F}, \phi) \subset M(\mathfrak{F}, \phi)^{\prime}
$$

from the following calculations.

$$
\begin{aligned}
& {\left[\pi(x), \pi^{\prime}(y)\right] }=\sum_{n} p_{n} \pi_{n}(x)\left(p_{n}^{*} p_{n}\right) \pi_{n}^{\prime}(y) p_{n}^{*} \\
&-\sum_{n} p_{n} \pi_{n}^{\prime}(y)\left(p_{n}^{*} p_{n}\right) \pi_{n}(x) p_{n}^{*} \\
&=\sum p_{n}\left[\pi_{n}(x), \pi_{n}^{\prime}(y)\right] p_{n}^{*}=0, \\
& {\left[U, \pi^{\prime}(y)\right]=\sum_{0}^{\infty} p_{n+1}[1, y] p_{n}^{*} } \\
&+\sum_{-\infty}^{-1} p_{n+1}\left\{V^{*} \phi^{\prime|n|}(1) \phi^{\prime|n|}(y)-\phi^{\prime|n|-1}(y) \phi^{\prime|n|-1}(1) V^{*}\right\} p_{n}^{*} \\
&=0,
\end{aligned}
$$




$$
\begin{aligned}
{\left[U^{\prime}, \pi(x)\right]=} & J(\mathfrak{\mho}, \phi)\left[U, \pi^{\prime}\left(j_{\Psi}(x)\right)\right] J(\mathfrak{\mho}, \phi)=0, \\
{\left[U, U^{\prime}\right]=} & \sum_{1}^{\infty} p_{n}\left\{p_{n-1}^{*} p_{n-1} V^{*}-V^{*} p_{n+1}^{*} p_{n+1}\right\} p_{n}^{*} \\
& +p_{0}\left\{V^{*} p_{-1}^{*} p_{-1}-V^{*} p_{1}^{*} p_{1}\right\} p_{0}^{*} \\
& +\sum_{-\infty}^{-1} p_{n}\left\{V^{*} p_{n-1}^{*} p_{n-1}-p_{n+1}^{*} p_{n+1} V^{*}\right\} p_{n}^{*} \\
= & 0, \\
{\left[U^{*}, U^{\prime}\right]=} & \sum_{2}^{\infty} p_{n-2}\left\{p_{n-1}^{*} p_{n-1} V^{*}-V^{*} p_{n-1}^{*} p_{n-1}\right\} p_{n}^{*}+p_{-1}\left\{V V^{*}-p_{0}^{*} p_{0}\right\} p_{1}^{*} \\
& +\sum_{-\infty}^{0} p_{n-2}\left\{V p_{n-1}^{*} p_{n-1}-p_{n-1}^{*} p_{n-1} V\right\} p_{n}^{*} \\
= & 0,
\end{aligned}
$$

where (2.4), (2.11), (2.8) and $p_{n} p_{k}^{*} p_{k}=p_{n}, p_{k}^{*} p_{k} p_{n}^{*}=p_{n}^{*}$ for $|n| \geqq|k|$, $n k \geqq 0$ are used.

From definitions, we have for $n \geqq 0$

$$
\pi(\mathfrak{F}) U^{* n} \Omega(\mathfrak{F}, \phi)=p_{-n} \mathfrak{F} D_{\phi}^{(n)} \Psi
$$

Since $s\left(D_{\phi}^{(n)}\right)=\phi^{n}(1)$, the closure of $p_{-n} \mathfrak{F} D_{\phi}^{(n)} \Psi$ contains

$$
\begin{aligned}
p_{-n} \mathfrak{\Im} \phi^{n}(1) \Psi & =p_{-n} \mathfrak{\Im} J_{\Psi} \phi^{n}(1) \Psi=p_{-n} j_{\Psi}\left\{\phi^{n}(1)\right\} \mathfrak{F} \Psi \\
& =p_{-n} \mathfrak{\mho} \Psi
\end{aligned}
$$

which is dense in $\mathfrak{S}_{-n}$. We also have for $n>0$,

$$
U^{n} \pi(\mathfrak{F}) \Omega(\mathfrak{F}, \phi)=p_{n} \mathfrak{F} \Psi
$$

which is dense in $\mathfrak{Q}_{n}$. Hence $\Omega(\mathfrak{F}, \phi)$ is cyclic for $M(\mathfrak{F}, \phi)$.

(2.29) and (2.30), together with (2.28), imply that $\Omega(\mathfrak{F}, \phi)$ is cyclic for $M^{\prime}(\mathfrak{F}, \phi)$ and hence separating for $M(\mathfrak{F}, \phi)$ by (2.31).

Setting $S=J(\mathfrak{F}, \phi) \Delta(\mathfrak{F}, \phi)^{1 / 2}$, we obtain for $n \geqq 0$

$$
S \pi(x) U^{* n} \Omega(\mathfrak{F}, \phi)=p_{n} J_{\Psi} x D_{\phi}^{(n)} j_{\Psi}\left(D_{\phi}^{(n)}\right)^{-1} \phi^{\prime n}(1) \Psi .
$$

Since $j_{\Psi}\left(D_{\phi}^{(n)}\right)^{-1} \phi^{\prime n}(1) \Psi=J_{\Psi}\left(D_{\phi}^{(n)}\right)^{-1} \phi^{n}(1) \Psi=\left(D_{\phi}^{(n)}\right)^{-1} \phi^{n}(1) \Psi$, we obtain 


$$
\begin{gathered}
S \pi(x) U^{* n} \Omega(\Im, \phi)=p_{n} J_{\Psi} x \phi^{n}(1) \Psi=p_{n} x * \Psi \\
=U^{n} \pi\left(x^{*}\right) \Omega(\Im, \phi) .
\end{gathered}
$$

From (2.24) and (2.25), $J(\mathfrak{F}, \phi) \Delta(\mathfrak{F}, \phi)=\Delta(\mathfrak{F}, \phi)^{-1} J(\mathfrak{F}, \phi)$. Hence $S^{2}=1$ whenever $S$ is defined. Hence

$$
S U^{n} \pi(x) \Omega(\mathfrak{J}, \phi)=\pi\left(x^{*}\right) U^{* n} \Omega(\mathfrak{F}, \phi) .
$$

Due to (2.4), we have

$$
\begin{aligned}
\pi(x) U^{*} & =\sum_{1}^{\infty} p_{n-1} \phi^{n}(x) p_{n}^{*}+\sum_{-\infty}^{0} p_{n-1} V x p_{n}^{*} \\
& =\sum_{1}^{\infty} p_{n-1} \phi^{n-1}(\phi(x)) p_{n}^{*}+\sum_{-\infty}^{0} p_{n-1} \phi(x) V p_{n}^{*} \\
& =\pi(\phi(x)) U^{*} \\
\pi(x) U & =\left\{U^{*} \pi\left(x^{*}\right)\right\}^{*}=\left\{\pi(\phi(x))^{*} U^{*}\right\}^{*} \\
& =U \pi(\phi(x)) .
\end{aligned}
$$

Due to (2.9) with $n=1$, we have

$$
\begin{aligned}
& \pi(x) U^{*}=\sum_{1}^{\infty} p_{n-1} \phi^{n-1}(x) p_{n}^{*}+\sum_{-\infty}^{0} p_{n-1} x V p_{n}^{*} \\
& =\sum_{1}^{\infty} p_{n-1} \phi^{n-1}(x \phi(1)) p_{n}^{*}+\sum_{-\infty}^{0} p_{n-1} x \phi(1) V p_{n}^{*} \\
& =\pi(x \phi(1)) U^{*}, \\
& U \pi(x)=\left(\pi\left(x^{*}\right) U^{*}\right)^{*}=U \pi(\phi(1) x), \\
& U \pi(x) U^{*}=\pi\left(\phi^{-1}\{\phi(1) x \phi(1)\}\right) U U^{*}, \\
& U U^{*} \Omega(\mathfrak{F}, \phi)=p_{0} V^{*} V \Psi \\
& =\Omega(\mathfrak{F}, \phi) \\
& U^{*} U \Omega(\mathfrak{F}, \phi)=p_{0} p_{1}^{*} p_{1} \Psi=\pi(\phi(1)) \Omega(\mathfrak{F}, \phi) .
\end{aligned}
$$

The last two equations imply

$$
U U^{*}=1, \quad U^{*} U=\pi(\phi(1)) .
$$


Therefore $\pi(F) U^{* n}$ and $U^{n} \pi(F)$ with varying $n$ together are total in $M(\mathfrak{F}, \pi)$. Hence

$$
\left(J(\mathfrak{F}, \phi) \Delta(\mathfrak{F}, \phi)^{1 / 2}\right) Q \Omega(\mathfrak{\jmath}, \phi)=Q^{*} \Omega(\mathfrak{F}, \phi)
$$

for all $Q \in M(\mathfrak{\mho}, \phi)$.

Let $D_{\phi}^{(n)}=\int L d E_{L}$ and $j_{\Psi}\left\{\left(D_{\phi}^{(n)}\right)^{-1} \phi^{\prime n}(1)\right\}=\int L d j_{\Psi}\left(E_{L}^{\prime}\right)$.

Since $E_{L} \mathfrak{F}$ and

$$
\mathfrak{F} E_{L}^{\prime} D_{\phi}^{(n)} \Psi=\mathfrak{F} D_{\phi}^{(n)} E_{L}^{\prime} \Psi=\mathfrak{F} D_{\phi}^{(n)} j_{\Psi}\left(E_{L}^{\prime}\right) \Psi=j_{\Psi}\left(E_{L}^{\prime}\right) \mathfrak{F} D_{\phi}^{(n)} \Psi
$$

are total sets of analytic vectors in $\phi^{n}(1) \Re$ and $\phi^{\prime n}(1) \Re$, respectively, $M(\mathfrak{F}, \phi) \Omega(\mathfrak{F}, \phi)$ is a core of $\Delta(\mathfrak{F}, \phi)^{1 / 2}$.

Hence $\Delta(\mathfrak{F}, \phi)$ and $J(\mathfrak{\mho}, \phi)$ are modular operator and modular conjugation operator for $\Omega(\mathfrak{F}, \phi)$.

By (2.29) and (2.30), we have

$$
M^{\prime}(\mathfrak{F}, \phi)=J(\mathfrak{F}, \phi) M(\mathfrak{F}, \phi) J(\mathfrak{F}, \phi)=M(\mathfrak{F}, \phi)^{\prime} .
$$

If $A_{\phi}>1$, then $\phi^{n}\left(A_{\phi}\right)>\phi^{n}(1)$ and hence $D_{\phi}^{(n)}>\phi^{n}(1)$. Then $\mathfrak{Q}_{0}$ is the eigenspace of $\Delta(\mathfrak{F}, \phi)$ belonging to 1 . If $Q \in M(\mathfrak{F}, \phi)$ is invariant under modular automorphisms for $\Omega(\mathfrak{F}, \phi)$, then $Q$ leaves $\mathfrak{S}_{0}$ invariant and hence the restriction of $Q$ to $\mathfrak{S}_{0}$ must be in the von Neumann algebra generated by $E_{0} M(\mathfrak{F}, \phi) E_{0}$ where $E_{0}$ is the projection on $\mathfrak{Q}_{0}$. Since $\pi(F) U^{* n}$ and $U^{n} \pi(F)$ are total in $M(\mathfrak{F}, \phi)$ and $E_{0} \pi(F) U^{* n} E_{0}=E_{0} U^{n} \pi(F) E_{0}=0$ for $n \neq 0$, we have $E_{0} M(\mathfrak{\mho}, \phi) E_{0}=\pi(\mathfrak{F}) E_{0}$. Hence $Q E_{0}=\pi(x) E_{0}$ for some $x \in \mathfrak{F}$. Since $\Omega(\widetilde{F}, \phi)$ is separating, $Q=\pi(x) \in \pi(\mathfrak{F}) E_{0}$. Conversely, all elements in $\pi(\mathfrak{F})$ commute with $\Delta(\mathfrak{F}, \phi)$, which is most easily demonstrated by

$$
\left\{\Delta(\mathfrak{F}, \phi)^{i t} \pi(x) \Delta(\mathfrak{F}, \phi)^{-i t}-\pi(x)\right\} \Omega(\mathfrak{F}, \phi)=0
$$

If $A_{\phi}=e^{a / 2}$, then $D_{\phi}^{(n)}=e^{n a / 2} \phi^{n}(1)$ and hence $\mathscr{S}_{n}$ is the eigenspace of $\Delta(\mathfrak{F}, \phi)$ belonging to an eigenvalue $e^{n a}$ and hence the spectrum of $\Delta(\mathfrak{F}, \phi)$ is $S_{x}, x=e^{a}$.

If $z$ is in the center of $M(\mathfrak{F}, \phi)$, then by a general result it is in the center of the set of elements in $M(\mathfrak{F}, \phi)$ commuting with $\Delta(\mathfrak{F}, \phi)$. Under the assumption $A_{\phi}>1, z=\pi(\bar{z}), \bar{z} \in \mathfrak{F}_{c}$. It is in the center of $M(\mathfrak{F}, \phi)$ if and only if 


$$
U \pi(\bar{z})=\pi(\bar{z}) U
$$

Since $U U^{*}=1$ and $U \pi(\bar{z}) U^{*}=\pi\left(\phi^{-1}\{\phi(1) \bar{z} \phi(1)\}\right)$, this condition is equivalent to $\phi(\bar{z})=\phi(1) \bar{z} \phi(1)=\bar{z} \phi(1)$. Hence the center of $M(\mathfrak{F}, \phi)$ consists of $\pi(z)$ such that $z \in \mathfrak{F}_{c}$ and $\phi(z)=z \phi(1)$.

If $\phi(z)=z \phi(1)$ for all $z \in \mathfrak{F}_{c}$, then

$$
(\Psi, \phi(z) \Psi)=(\Psi, z \phi(1) \Psi)=\left(\Psi, z \phi(1)^{\natural} \Psi\right) .
$$

Since $\phi$ and $\hbar$ are faithful, $\phi(z)^{\natural}=z \phi(1)^{\natural} \neq 0$ for $z \in \mathfrak{F}_{c}, z \neq 0$. Hence $s\left(\phi(1)^{\natural}\right)=1$ and $\left(\phi(1)^{\natural}\right)^{-1}$ exists. Then

$$
(\Psi, \phi(A z A) \Psi)=(\Psi, z \Psi)
$$

if $A=\left\{\phi(1)^{\natural}\right\}^{-1 / 2}$. Since such $A$ in $\mathfrak{F}_{c}$ is unique, we have $A_{\phi}=\left(\phi(1)^{\natural}\right)^{-1 / 2}$.

Remark 1. If $\phi(z)=z \phi(1)$ for all $z \in \mathfrak{F}_{c}$, then the condition $A_{\phi}>1$ is equivalent to the condition that $\phi$ is a proper injective endomorphism of $z \mathfrak{F}$, for every central projection $z \neq 0$, as is seen by the following argument.

If $A_{\phi}=1$, then $(\Psi,(1-\phi(1)) \Psi)=0$. Since $\Psi$ is separating, $\phi(1)=1$ and $\phi$ is an automorphism. If $p$ is the projection on the eigenspace of $A_{\phi}$ belonging to 1 , then the same argument shows $p=\phi(p)$ and hence $\phi$ is an automorphism on $p \mathfrak{F}$. Since $p \geqq p \phi(1)=\phi(p)$ for every central projection, $A_{\phi} \geqq 1$ and hence $A_{\phi}>1$ is equivalent to $\phi$ not being an automorphism on $z \mathfrak{F}$ for every central projection $z \neq 0$.

Remark 2. If $\mathfrak{₹}$ is commutative and $\phi$ is an automorphism, then $M(\mathfrak{F}, \phi)$ is the von Neumann algebra obtained by group-measure construction with the additive group of integers.

\section{§3. Proof of Theorem 1}

We consider the von Neumann algebra $\mathfrak{M}$ on a space $\mathfrak{L}$ in Theorem 1. We start with preliminary analysis.

In [3], we have considered the set $\mathfrak{M l}_{J}$ of operators $Q$ in $\mathfrak{M}$ satisfying $Q \mathfrak{S}(I) \subset \mathfrak{S}(I+J)$ for every finite open interval $I$ where $\mathfrak{S}((\alpha, \beta))=$ 
$\left(E_{\beta-0}-E_{\alpha+0}\right) H, E_{\lambda}$ is the spectral projection of $\log \Delta=\int \lambda d E_{\lambda}$ and $J$ is a finite open interval. We assume that the spectrum of $\log \Delta$ is $\left\{0, \pm \log x_{1}\right.$, $\left.\pm \log x_{2}, \ldots\right\}$.

If the spectrum of $\log \Delta$ contained in $J$ is just one point $\alpha$, then $\mathfrak{g}(J)=\mathfrak{S}_{\alpha}$. Let $Q \in \mathfrak{M}_{J}$ for such $J$. Then

$$
\{\tau(t) Q\} \Omega=\Delta^{\imath t} Q \Omega=e^{i \alpha t} Q \Omega .
$$

Since $\Omega$ is separating, $\tau(t) Q=e^{i \alpha t} Q$ and $Q \in \mathfrak{M}_{\alpha}$.

Hence $\mathfrak{M}_{J}=\mathfrak{M}_{\alpha}$.

By Lemma 7, Lemma 5 and (2.1) of [3], we have

$$
\begin{aligned}
& \mathfrak{M}_{\alpha} \mathfrak{M}_{\beta} \subset \mathfrak{M}_{\alpha+\beta}, \\
& \mathfrak{M}_{\alpha}^{*}=\mathfrak{M}_{-\alpha}, \\
& \overline{\mathfrak{M}_{\alpha} \Omega}=\mathfrak{L}_{\alpha} .
\end{aligned}
$$

Lemma 5. $\cup_{\alpha} \mathfrak{M}_{\alpha}$ is total in $\mathfrak{M}$.

Proof. Let $J_{n}=\left(\log x_{n-1}, \log x_{n+1}\right), n=0, \pm 1, \ldots$ where $x_{0}=1$. Let $\tilde{\phi}_{n}, n=0, \pm 1, \ldots$ be non-negative $C^{\infty}$ functions with a compact support in $J_{n}$ such that $\Sigma \tilde{\phi}_{n}=1$. Let $\tilde{f}$ be any $C^{\infty}$ function with a compact support. Let

$$
\begin{aligned}
& f(t)=(2 \pi)^{-1} \int \tilde{f}(\lambda) e^{-i \lambda t} d \lambda \\
& f_{n}(t)=(2 \pi)^{-1} \int \tilde{f}(\lambda) \tilde{\phi}_{n}(\lambda) e^{-i \lambda t} d \lambda
\end{aligned}
$$

Then $f=\sum_{n} f_{n}$ (finite sum) and $Q\left(f_{n}\right) \in \mathfrak{M}_{\log x_{n}}$ by Lemma 6 of [3]. Hence $Q(f) \in \cup_{\alpha} \mathfrak{M}_{\alpha}$.

Let $\tilde{g}$ be a non-negative $C^{\infty}$ function with a compact support such that $\tilde{g}(0)=1$ and $g(t)=(2 \pi)^{-1} \int e^{-i \lambda t} \tilde{g}(\lambda) d \lambda$. Let $g_{n}(t)=n g(n t)$ and $\tilde{g}_{n}(\lambda)=\tilde{g}(\lambda / n)$. It is then easy to see $\lim _{n \rightarrow \infty} Q\left(g_{n}\right)=Q$. Since $Q\left(g_{n}\right) \in \cup_{\alpha} \mathfrak{M}_{n}$, we have the lemma. 
Lemma 6. $\overline{\left(\mathfrak{M i}_{\alpha}\right)_{p . i .} \mathfrak{S}_{0}}=\mathfrak{L}_{\alpha}$, where $\left(\mathfrak{M U}_{\alpha}\right)_{p . i \text {. }}$ is the set of all partial isometries in $\mathfrak{M q}_{\alpha}$ and the bar denotes the strong closure.

Proof. Let $\Psi \in \mathfrak{L}_{\alpha}$ and $\varepsilon>0$ be given. By (3.3), there exists $Q \in \mathfrak{M}_{\alpha}$ such that $\|Q \Omega-\Psi\|<\varepsilon / 2$.

Let $Q^{*} Q=\int \lambda d e_{\lambda}$. Since $Q^{*} Q \in \mathfrak{M}_{0}$ by (3.1) and (3.2) and since $\mathfrak{M}_{0}$ is a von Neumann algebra, $e_{\lambda} \varepsilon \mathfrak{M}_{0}$. Let $\delta>0$ be such that $Q_{\delta}=Q\left(1-e_{\delta}\right)$ satisfies $\left\|Q_{\delta} \Omega-Q \Omega\right\|<\varepsilon / 2$. Let $|Q|_{\delta}=\left(Q^{*} Q\right)^{1 / 2}\left(1-e_{\delta}\right)+e_{\delta}$. Then $|Q|_{\delta}$ has a bounded inverse $|Q|_{\delta}^{-1} \in \mathfrak{M}_{0}, U=Q_{\delta}|Q|_{\delta}^{-1} \in\left(\mathfrak{M}_{\alpha}\right)_{p . i .}, \Phi \equiv|Q|_{\delta} \Omega \in \mathfrak{S}_{0}$ and $U \Phi=Q_{\delta} \Omega$ satisfies $\|U \Phi-\Psi\|<\varepsilon$.

We need a Lemma on the mapping $F_{\rho}^{Z R}$ introduced in [2].

Lemma 7. If $Z$ is a center of a finite von Neumann algebra $R$ and $\rho$ is a trace on $R$, then

$$
F_{\rho}^{Z R}(Q)=Q^{\natural} s^{Z}(\rho)
$$

Proof. As proved in $[2], F_{\rho}^{Z R}$ is $Z$-linear, positive normal mapping from $R$ onto $Z s^{Z}(\rho)$, vanishing on $\left(1-s^{Z}(\rho)\right) R+R\left(1-s^{Z}(\rho)\right)$ and strictly positive on $s^{Z}(\rho) R$. Since $\rho$ is a trace state, we have

$$
\rho\left(Q_{1} Q_{2} z\right)=\rho\left(Q_{1} z Q_{2}\right)=\rho\left(Q_{2} Q_{1} z\right)
$$

for $z \in Z$. Hence $F_{\rho}^{Z R}\left(Q_{1} Q_{2}\right)=F_{\rho}^{Z R}\left(Q_{2} Q_{1}\right)$, which implies $F_{\rho}^{Z R}\left(U Q U^{*}\right)=$ $F_{\rho}^{Z R}(Q)$ for all unitary $U$ in $R$. Hence $F_{\rho}^{Z R}$ is the canonical mapping of $R s^{Z}(\rho)$ and we have (3.4).

Q.E.D.

\section{Proof of Theorem 1}

Step 1. Let be $s_{\alpha}$ the support of $\mathfrak{L}_{\alpha}$ in $\mathfrak{M}$, namely the smallest projection in $\mathfrak{M}$ satisfying $\left(1-s_{\alpha}\right) \mathfrak{S}_{\alpha}=0$. We prove $s_{\alpha} \in \mathbb{Z}$.

Since $\mathfrak{S}_{\alpha}$ is invariant under $\Delta^{i t}$ (as a set), $\left[s_{\alpha}, \Delta^{i t}\right]=0$ for all real $t$. Hence $s_{\alpha} \in \mathfrak{M R}_{0}$.

Since $\mathfrak{M}_{0} \mathfrak{S}_{\alpha}=\mathfrak{S}_{\alpha}$, any $Q \in \mathfrak{M}_{0}$ commutes with $s_{\alpha}$. Hence $s_{\alpha} \in \mathfrak{Z}_{0}$.

By assumption $3=Z_{0}$, we have $s_{\alpha} \in 3$.

Step 2. $\sup \left\{U U^{*} ; U \in\left(\mathfrak{M}_{\alpha}\right)_{p . i .}\right\}=s_{\alpha}$. 
Since $U \Omega \in \mathfrak{S}_{\alpha},\left(1-s_{\alpha}\right) U \Omega=0$ which implies $\left(1-s_{\alpha}\right) U=0$. Hence $s_{\alpha} \geqq U U^{*}$ for all $U \in\left(\mathfrak{M}_{\alpha}\right)_{p . i .}$.

By Lemma 6, $\left\{U \mathfrak{S}_{0}\right\}$ is dense in $\mathfrak{S}_{\alpha}$. Hence

$$
\left\{U U^{*} \mathfrak{g}\right\}=\{U \mathfrak{S}\}=\left\{\left(U_{\mathfrak{M}^{\prime}} \mathfrak{E}_{0}\right)^{-}\right\}=\left\{\left(\mathfrak{M}^{\prime} U \mathfrak{S}_{0}\right)^{-}\right\}
$$

is dense in $\left(\mathfrak{M}^{\prime} \mathfrak{S}_{\alpha}\right)^{-}=s_{\alpha} \mathfrak{W}$.

Step 3. $\sup \left\{U^{*} U ; U \in\left(\mathfrak{M}_{\alpha}\right)_{p . i .}\right\}=s_{\alpha}$.

Since $J \Delta=\Delta^{-1} J$, we have $J \mathfrak{E}_{\alpha}=\mathfrak{S}_{-\alpha}$. Hence $s_{-\alpha}=j\left(s_{\alpha}^{\prime}\right)$ where $s_{\alpha}^{\prime}$ is the support of $\mathfrak{S}_{\alpha}$ relative to $\mathfrak{M}^{\prime}$. Since $s_{-\alpha} \in Z$ by Step $1, s_{\alpha}^{\prime}=j\left(s_{-\alpha}\right)=$ $s_{-\alpha} \in Z$. We now have $\mathfrak{S}_{\alpha} \subset s_{\alpha}^{\prime} \mathfrak{W}$ and hence $s_{\alpha} \mathfrak{W}=\overline{\mathfrak{M}^{\prime} \mathfrak{S}_{\alpha}} \subset s_{\alpha}^{\prime} \mathfrak{W}$, which implies $s_{\alpha} \leqq s_{\alpha}^{\prime}$. At the same time $\mathfrak{L}_{\alpha} \subset s_{\alpha} \mathfrak{S}$. Hence $s_{\alpha}^{\prime} \mathfrak{S}=\overline{\mathfrak{M} \mathfrak{S}_{\alpha}} \subset s_{\alpha} \mathfrak{S}$ which implies $s_{\alpha}^{\prime} \leqq s_{\alpha}$. Hence $s_{\alpha}=s_{\alpha}^{\prime}=s_{-\alpha}$. By (3.2) and Step 2, we obtain Step 3 .

Step 4. For $U \in\left(\mathfrak{M}_{\alpha}\right)_{p . i .}$.

$$
\left(U U^{*}\right)^{\natural}=e^{\alpha}\left(U^{*} U\right)^{\natural} .
$$

Proof. For $z \in 3_{0}$, we have

$$
\begin{aligned}
\left(\Omega, U U^{*} z \Omega\right) & =\left(\Omega, U z U^{*} \Omega\right) \quad\left(\text { by } 3=\mathfrak{Z}_{0}\right) \\
& =\left(\Omega, U z J \Delta^{1 / 2} U \Omega\right) \\
& =(\Omega, U z j(U) \Omega) e^{\alpha / 2} \quad\left(\text { by } U \Omega \in \mathfrak{S}_{\alpha}\right) \\
& =\left(j\left(U^{*}\right) \Omega, U z \Omega\right) e^{\alpha / 2} \\
& =\left(\Omega, U^{*} U z \Omega\right) e^{\alpha} .
\end{aligned}
$$

Hence

$$
F_{\omega_{g}}^{B_{0} \mathfrak{M}_{0}}\left(U U^{*}\right)=e^{\alpha} F_{\omega_{g}}^{B_{0} \mathfrak{M}_{0}}\left(U^{*} U\right) .
$$

By Lemma 7, we have (3.5).

Step 5. There exists $U_{\alpha} \in\left(\mathfrak{M}_{\alpha}\right)_{p . i}$. such that $U_{\alpha} U_{\alpha}^{*}=s_{\alpha}$ for $\alpha>0$.

Consider a maximal family of $U_{\nu} \in\left(\mathfrak{M}_{\alpha}\right)_{p . i}$. such that $U_{\nu} U_{\nu}^{*}$ are mutually orthogonal and $U_{\nu}^{*} U_{\nu}$ are mutually orthogonal. Then $U_{\alpha} \equiv \Sigma U_{\nu}$ $\in\left(\mathfrak{M}_{\alpha}\right)_{p . i .}$. Assume $s_{\alpha}-U_{\alpha} U_{\alpha}^{*} \neq 0$. By Step 2, there exists $U_{(0)} \in\left(\mathfrak{M}_{\alpha}\right)_{p . i .}$. 
such that $c\left(s_{\alpha}-U_{\alpha} U_{\alpha}^{*}\right) U_{(0)} U_{(0)}^{*} \neq 0$ where $c(\cdot)$ denotes the central support. By the comparability theorem, there exists a projection $e$ in $Z_{0}$ and partial isometries $u_{1}$ and $u_{2}$ in $\mathfrak{M}_{0}$ such that $u_{1}^{*} u_{1}=e\left(s_{\alpha}-U_{\alpha} U_{\alpha}^{*}\right), u_{1} u_{1}^{*} \leqq$ $e U_{(0)} U_{(0)}^{*}, \quad u_{2}^{*} u_{2}=(1-e)\left(U_{(0)} U_{(0)}^{*}\right)$ and $u_{2} u_{2}^{*} \leqq(1-e)\left(s_{\alpha}-U_{\alpha} U_{\alpha}^{*}\right)$. Due to $c\left(s_{\alpha}-U_{\alpha} U_{\alpha}^{*}\right) U_{(0)} U_{(0)}^{*} \neq 0$, we have either $u_{1} \neq 0$ or $u_{2} \neq 0$. If $u_{1} \neq 0$, we set $U^{\prime}=e u_{1}^{*} U_{(0)}$. Then $U^{\prime} U^{*}=u_{1}^{*} u_{1} \leqq s_{\alpha}-U_{\alpha} U_{\alpha}^{*}, U^{\prime} \neq 0$ and $U^{\prime} \varepsilon\left(M_{\alpha}\right)_{p . i .}$. If $u_{2} \neq 0$, we set $U^{\prime}=(1-e) u_{2} U_{(0)}$. Then $U^{\prime} U^{\prime *}=u_{2} u_{2}^{*} \leqq s_{\alpha}-U_{\alpha} U_{\alpha}^{*}$, $U^{\prime} \neq 0$ and $U^{\prime} \varepsilon\left(\mathfrak{M}_{\alpha}\right)_{p . i .}$.

By Step 4,

$$
\begin{aligned}
\left(s_{\alpha}-U_{\alpha}^{*} U_{\alpha}\right)^{\natural}= & s_{\alpha}-e^{-\alpha}\left(U_{\alpha} U_{\alpha}^{*}\right)^{\natural} \\
& \geqq e^{-\alpha}\left(s_{\alpha}-U_{\alpha} U_{\alpha}^{*}\right)^{\natural} \\
& \geqq e^{-\alpha}\left(U^{\prime} U^{\prime *}\right)^{\natural}=\left(U^{\prime *} U^{\prime}\right)^{\natural} .
\end{aligned}
$$

Hence there exists $u \in\left(\mathfrak{M}_{0}\right)_{p, i}$. such that

$$
u u^{*}=U^{\prime *} U^{\prime}, u^{*} u \leqq s_{\alpha}-U_{\alpha}^{*} U_{\alpha} .
$$

Setting $U^{\prime \prime}=U^{\prime} u$, we have $U^{\prime \prime} \in\left(\mathfrak{M}_{\alpha}\right)_{p . i}$. and

$$
\begin{aligned}
& U^{\prime \prime} * U^{\prime \prime}=u^{*} u \leqq s_{\alpha}-U_{\alpha}^{*} U_{\alpha}, \\
& U^{\prime \prime} U^{\prime \prime *}=U^{\prime} U^{\prime *} \leqq s_{\alpha}-U_{\alpha} U_{\alpha}^{*} .
\end{aligned}
$$

We also have $U^{\prime \prime} U^{\prime \prime *}=U^{\prime} U^{*} \neq 0$ and hence $U^{\prime \prime} \neq 0$. This contradicts with the maximality of $\left\{U_{\nu}\right\}$.

Step 6. Fix $U_{\alpha}$ such that $U_{\alpha} \in\left(\mathfrak{M}_{\alpha}\right)_{p . i .}, U_{\alpha} U_{\alpha}^{*}=s_{\alpha}$. Then $\left(U_{\alpha}^{*} U_{\alpha}\right)^{\natural}=$ $e^{-\alpha} s_{\alpha}$. Such $U_{\alpha}$ exists for $\alpha>0$ by Step 5. Define an injective endomorphism $\phi_{\alpha}$ of $s_{\alpha} \mathfrak{M R}_{0}$ by $\phi_{\alpha}(Q)=U_{\alpha}^{*} Q U_{\alpha}$. Then

$$
\begin{gathered}
\phi_{\alpha}\left(\mathfrak{M}_{0}\right)=\phi_{\alpha}(1) \mathfrak{M}_{0} \phi_{\alpha}(1), \\
\phi_{\alpha}(z)=z \phi_{\alpha}(1), z \in \mathfrak{Z}_{0}, \\
\phi_{\alpha}(1)^{\natural}=e^{-\alpha} s_{\alpha} .
\end{gathered}
$$

(3.8) is the same as $\left(U_{\alpha}^{*} U_{\alpha}\right)^{\natural}=e^{-\alpha} s_{\alpha}$. (3.7) follows from $3_{0}=3$, which implies $U_{\alpha}^{*} z U_{\alpha}=z U_{\alpha}^{*} U_{\alpha}=z \phi_{\alpha}(1)$ if $z \in 3_{0}$. Let $e$ be a projection 
in $\phi_{\alpha}(1) \mathfrak{M}_{0} \phi_{\alpha}(1)$. Then $e \leqq \phi_{\alpha}(1)=U_{\alpha}^{*} U_{\alpha}$. Let $e^{\prime}=U_{\alpha} e U_{\alpha}^{*} \in \mathfrak{M}_{0}$. Then $e=U_{\alpha}^{*} e^{\prime} U_{\alpha}=\phi_{\alpha}\left(e^{\prime}\right)$. Hence $\phi_{\alpha}(1) \mathfrak{M}_{0} \phi_{\alpha}(1) \subset \phi_{\alpha}\left(\mathfrak{M}_{0}\right)$. Conversely $\phi_{\alpha}\left(\mathfrak{M}_{0}\right) \subset$ $\phi_{\alpha}(1) \phi_{\alpha}\left(\mathfrak{M R}_{0}\right) \phi_{\alpha}(1) \subset \phi_{\alpha}(1) \mathfrak{M}_{0} \phi_{\alpha}$ (1). Hence (3.6) holds.

$\phi_{\alpha}$ is injective on $\mathfrak{M}_{0} s_{\alpha}$ because $\phi_{\alpha}(Q)=0$ implies $0=U_{\alpha} \phi_{\alpha}(Q) U_{\alpha}^{*}=$ $s_{\alpha} Q s_{\alpha}=Q$ for $Q \in s_{\alpha} \mathfrak{M}_{0} . \quad \phi_{\alpha}$ is a $*$ homomorphism because $\phi_{\alpha}\left(Q_{1}\right) \phi_{\alpha}\left(Q_{2}\right)=$ $\phi_{\alpha}\left(Q_{1} s_{\alpha} Q_{2}\right)=\phi_{\alpha}\left(Q_{1} Q_{2}\right)$ for $Q_{1}, Q_{2} \in s_{\alpha} \mathfrak{M}_{0}$ and $\phi_{\alpha}(Q)^{*}=\phi_{\alpha}\left(Q^{*}\right)$.

Step 7. $s_{\alpha}\left(\mathfrak{M}_{n \alpha}\right)_{p . i .} \subset U_{\alpha}^{n}\left(\mathfrak{M}_{0}\right)_{p . i .}$ for $n \geqq 0$ and $s_{\alpha}\left(\mathfrak{M}_{n \alpha}\right)_{p, i .} \subset\left(\mathfrak{M}_{0}\right)_{p . i .}$ $U_{\alpha}^{* n}$ for $n \leqq 0$, where $U_{\alpha}$ is from Step 6 .

Let $n>0$ and $V \in s_{\alpha}\left(\mathfrak{M}_{n \alpha}\right)_{p . i .} . \quad$ By (3.1) and (3.2), $w=U_{\alpha}^{*_{n}} V \in\left(\mathfrak{M}_{0}\right)$ and $U_{\alpha}^{n} w=s_{\alpha} V=V . \quad w$ is a partial isometry because $w w^{*}=\phi_{\alpha}^{n}\left(V V^{*}\right)$ and $w^{*} w=s_{\alpha} V^{*} V$ are both projections. Hence $s_{\alpha}\left(\mathfrak{M}_{n \alpha}\right)_{p . i .} \subset U_{\alpha}^{n}\left(\mathfrak{M}_{0}\right)_{p . i .}$. Taking adjoint and using (3.2), we have $s_{\alpha}\left(\mathfrak{M}_{n \alpha}\right)_{p . i .} \subset\left(\mathfrak{M}_{0}\right)_{p . i .} U_{\alpha}^{*|n|}$ for $n<0$. The case $n=0$ is trivial.

Step 8. Let $\left\{0, \pm a_{1}, \pm a_{2}, \ldots\right\}$ be spectrum of $\log \Delta$ such that $0<a_{1}<$ $a_{2}<\cdots$. Let

$$
\begin{gathered}
P_{n}=s_{a_{n}} \prod_{k=1}^{n-1}\left(1-s_{a_{k}}\right), n=1,2, \ldots, \\
P_{0}=1-\sum_{n=1}^{\infty} P_{n} .
\end{gathered}
$$

By definition and Step $1, P_{n}$ are mutually orthogonal central projections with the sum $\sum_{k=0}^{\infty} P_{k}=1 . \quad P_{1}=s_{a_{1}} \neq 0$. On $P_{0} \mathfrak{S}, \Delta$ has no eigenvalue larger than 1 and hence $\Delta=1$. (Each $P_{n} \mathfrak{S}$ is invariant under $J$ and $\Delta$ because $P_{n} \in 3$.) Consequently, $P_{0} \Omega$ is a cyclic and separating trace vector for $P_{0} \mathfrak{M}$.

Let $U_{n}=P_{n} U_{a_{n}}, \Omega_{n}=P_{n} \Omega, \mathfrak{F}_{n}=P_{n} \mathfrak{M}_{0} . \quad \mathfrak{F}_{n}$ is known to be a finite von Neumann algebra with $\Omega_{n}$ as a cyclic (in $P_{n} \mathfrak{S}$ ) and separating trace vector. Let $\phi_{n}=\phi_{a_{n}} \mid \mathfrak{F}_{n}$. It satisfies (3.6) (3.8), where $\phi_{\alpha}$ is replaced by $\phi_{n}, \mathfrak{M}_{0}$ by $\mathfrak{F}_{n}, \mathfrak{B}_{0}$ by the center $\mathfrak{F}_{n c}$ of $\mathfrak{F}_{n}, \alpha$ by $a_{n}$, and $s_{\alpha}$ by $P_{n}$.

Step 9. $\quad U_{n}^{m} \widetilde{\mho}_{n} \Omega_{n}$ and $\mathfrak{F}_{n}\left(U_{n}^{*}\right)^{m} \Omega_{n}, m=0,1,2, \ldots$ span the whole space $P_{n} \mathfrak{S}$.

By Step 7 and (3.3), they span $\sum_{m=-\infty}^{\infty} P_{n} \mathfrak{S}_{m a_{n}}$. Assume that $P_{n} \mathfrak{S}_{b} \neq 0$, $b \neq m a_{n}$ for any integer $m$. Since $P_{n} \mathfrak{E}_{-b}=P_{n} J \mathfrak{S}_{b}=J P_{n} \mathfrak{S}_{b} \neq 0$, we may 
assume $b>0$. There exists a non-negative integer $m$ such that $m a_{n}<b<$ $(m+1) a_{n}$.

$$
U_{n}^{m}\left(U_{n}^{m}\right)^{*}=P_{n} \geqq U_{b}^{\prime} U_{b}^{\prime *}
$$

for $U_{b}^{\prime}=P_{n} U_{b}$. We define $W=\left(U_{n}^{m}\right)^{*} U_{b}^{\prime} . \quad W \in P_{n} \mathfrak{M}_{b-m a_{n}}$. We also have

$$
W^{*} W=U_{b}^{\prime *} P_{n} U_{b}^{\prime}=U_{b}^{\prime *} U_{b}^{\prime} \neq 0 .
$$

Hence $0 \neq W \Omega_{n} \in P_{n} \mathfrak{S}_{b-m a_{n}}$. Since $0<b-m a_{n}<a_{n}$, this contradicts the definition of $P_{n}$.

Step 10. $P_{n} \mathfrak{M}, \Omega_{n}, \widetilde{\mho}_{n}$ and $U_{n}$ are unitarily equivalent to $M\left(\mathfrak{F}_{n}, \phi_{n}\right)$, $\Omega\left(\mathfrak{F}_{n}, \phi_{n}\right), \pi\left(\mathfrak{F}_{n}\right)$ and $U$ of Section 2 , where $\Psi$ is to be taken equal to $\Omega_{n}$.

$U_{n}^{m} \widetilde{\mho}_{n} \Omega_{n}, \quad m=0,1, \ldots \quad$ and $\mathfrak{\Im}_{n}\left(U_{n}^{*}\right)^{m \prime} \Omega_{n}, m^{\prime}=1,2, \ldots$ are orthogonal family of subspaces of $P_{n} \mathfrak{E}$ because they belong to different eigenvalues of $\Delta$. They span the whole space $P_{n} \mathfrak{S}$ by Step 9 .

We have for $x, y \in \mathfrak{F}_{n}$ and $m \geqq 0$,

$$
\begin{aligned}
\left(U_{n}^{m} x \Omega_{n}, U_{n}^{m} y \Omega_{n}\right) & =\left(\Omega_{n}, x * \phi_{n}^{m}(1) y \Omega_{n}\right) \\
& =\left(U^{m} \pi(x) \Omega\left(\mathfrak{\mho}_{n}, \phi_{n}\right), U^{m} \pi(y) \Omega\left(\mathfrak{\mho}_{n} \phi_{n}\right)\right)
\end{aligned}
$$

where we have used $\left(U^{*}\right)^{m} U^{m}=\pi\left(\phi_{n}^{m}(1)\right)$ which follows from (2.32) and $U^{*} \pi(x) U=\pi(\phi(x))$. For $m>0$,

$$
\left(x\left(U_{n}^{*}\right)^{m} \Omega_{n}, y\left(U_{n}^{*}\right)^{m} \Omega_{n}\right)=\left(\Omega_{n}, U_{n}^{m} x^{*} y\left(U_{n}^{*}\right)^{m} \Omega_{n}\right) .
$$

Since $U_{n} \phi_{n}(1)=U_{n} U_{n}^{*} U_{n}=U_{n}, U_{n}^{m}=U_{n} \phi_{n}(1) U_{n}^{m-1}=U_{n}^{m} \phi_{n}^{m}(1)$.

Hence $U_{n}^{m} x^{*} y\left(U_{n}^{*}\right)^{m}=U_{n}^{m} \phi_{n}^{m}(1) x^{*} y \phi_{n}^{m}(1)\left(U_{n}^{*}\right)^{m}$. There exists $Q \in \mathfrak{F}_{n}$ such that $\phi_{n}^{m}(Q)=\phi_{n}^{m}(1) x^{*} y \phi_{n}^{m}(1)$. Then $U_{n}^{m} \phi_{n}^{m}(Q)\left(U_{n}^{*}\right)^{m}=U_{n}^{m}\left(U_{n}^{*}\right)^{m} Q U_{n}^{m}\left(U_{n}^{*}\right)^{m}$ $=P_{n} Q P_{n}=Q$. Hence

$$
\begin{aligned}
\left(x\left(U_{n}^{*}\right)^{m} \Omega_{n}, y\left(U_{n}^{*}\right)^{m} \Omega_{n}\right) & =\left(\Omega_{n}, \phi_{n}^{-m}\left\{\phi_{n}^{m}(1) x^{*} y \phi_{n}^{m}(1)\right\} \Omega_{n}\right) \\
& =\left(\Omega\left(\mathfrak{F}_{n}, \phi_{n}\right), U^{m} \pi\left(x^{*} y\right) U^{* m} \Omega\left(\mathfrak{F}_{n}, \phi_{n}\right)\right) \\
& =\left(\pi(x) U^{* m} \Omega\left(\mathfrak{F}_{n}, \phi_{n}\right), \pi(y) U^{* m} \Omega\left(\mathfrak{F}_{n}, \phi_{n}\right)\right) .
\end{aligned}
$$

Therefore there exists a unitary mapping $p$ from $\mathfrak{S}$ of Section 2 to $P_{n} \mathfrak{S}$ such that for all $x \in \mathfrak{F}_{n}$, 


$$
\begin{aligned}
& p U^{m} \pi(x) \Omega\left(\mathfrak{F}_{n}, \phi_{n}\right)=U_{n}^{m} x \Omega_{n}, m=0,1, \ldots, \\
& p \pi(x) U^{* m} \Omega\left(\mathfrak{F}_{n}, \phi_{n}\right)=x\left(U_{n}^{*}\right)^{m} \Omega_{n}, m=1,2, \ldots
\end{aligned}
$$

It is then immediately seen that

$$
\begin{aligned}
& p^{*} x p=\pi(x), \\
& p^{*} U_{n} p=U .
\end{aligned}
$$

By Lemma 5 and Step $7, \mathfrak{F}_{n}$ and $U_{n}$ generate $\mathfrak{M P} P_{n}$. Hence $\mathfrak{M} P_{n}=$ $p M\left(\mathfrak{F}_{n}, \phi_{n}\right) p^{*}$.

Q.E.D.

\section{§4. Isomorphism among $M(\mathfrak{F}, \phi)$}

Theorem 4. Different choices of cyclic and separating trace vector $\Psi$ yield unitarily equivalent $M(\mathfrak{F}, \phi)$.

Proof. Let $\rho^{\prime}$ be another faithful tracial state of $\mathfrak{F}$. Then there exists a strictly positive selfadjoint operator $\alpha$ affiliated with $\mathfrak{F}_{c}$ such that the vector state by $\Psi^{\prime}=\alpha \Psi$ is $\rho^{\prime}$. It is sufficient to show that $P_{0} \Psi^{\prime}, \pi(\mathfrak{F})$ and $U$ have exactly the same structure as in the construction in section 2 where $\Psi$ is to be replaced by $\Psi^{\prime}$.

Let $A_{\phi}^{\prime}=\int \lambda d E_{\lambda}^{A^{\prime}}, E_{\lambda}^{A^{\prime}} \in \mathfrak{F}_{c}$ be defined similar to $A_{\phi}$ :

$$
\left(\phi\left(A_{\phi}^{\prime}\right) \Psi^{\prime}, \phi(z) \phi\left(A_{\phi}^{\prime}\right) \Psi^{\prime}\right)=\left(\Psi^{\prime}, z \Psi^{\prime}\right), \quad z \in \mathfrak{F}_{c} .
$$

Let $\alpha=\int \lambda d E_{\lambda}^{\alpha}, \alpha_{L}=\alpha E_{L}^{\alpha}$. Then

$$
\left\|z_{1} \alpha_{L} \Psi\right\|^{2}=\left\|\phi\left(z_{1} \alpha_{L} A_{\phi}\right) \Psi\right\|^{2}, \quad z_{1} \in \mathfrak{F}_{c}
$$

Hence $\phi\left(A_{\phi}\right) \Psi$ is in the domain of $\alpha$ and

$$
\left(\Psi^{\prime}, z^{\prime \prime}\right)=\left(\phi(\alpha) \phi\left(A_{\phi}\right) \Psi, \phi(z) \phi(\alpha) \phi\left(A_{\phi}\right) \Psi\right)
$$

for positive $z$ in $\mathfrak{F}_{c}$ and hence for any $z$ in $\mathfrak{F}_{c}$. Since $\alpha A_{\phi}$ and $\alpha^{-1}$ are strictly positive, $\phi\left(\alpha A_{\phi}\right) \alpha^{-1}$ is strictly positive on $\phi(1) \Re$ and hence there exists a positive selfadjoint operator

$$
A^{\prime}=\int \lambda_{1} \lambda_{2} \lambda_{3}^{-1} d E_{\lambda_{1}}^{\alpha} d E_{\lambda_{2}}^{A} d\left\{\phi^{-1}\left(E_{\lambda_{3}}^{\alpha} \phi(1)\right)\right\}
$$


affiliated with $\mathfrak{F}_{c}$ such that $\phi\left(A^{\prime}\right)=\phi\left(\alpha A_{\phi}\right) \alpha^{-1}$ and hence

$$
\left(\Psi^{\prime}, z \Psi^{\prime}\right)=\left(\phi\left(A^{\prime}\right) \Psi^{\prime}, \phi(z) \phi\left(A^{\prime}\right) \Psi^{\prime}\right)
$$

From the uniqueness of $A_{\phi}^{\prime}=(\text { Radon-Nikodym derivative })^{1 / 2}$, we have $A_{\phi}^{\prime}=A^{\prime}$ and

$$
\phi\left(A_{\phi}^{\prime}\right) \alpha=\phi\left(\alpha A_{\phi}\right)
$$

Hence

$$
\begin{aligned}
V Q \Psi^{\prime} & =\lim _{L} V Q \alpha_{L} \Psi \\
& =\lim _{L} \phi\left(Q \alpha_{L}\right) \phi\left(A_{\phi}\right) \Psi \\
& =\phi(Q) \phi\left(\alpha A_{\phi}\right) \Psi=\phi(Q) \phi\left(A_{\phi}^{\prime}\right) \Psi^{\prime}
\end{aligned}
$$

This shows that $V$ for $\Psi^{\prime}$ is the same as $V$ for $\Psi$. Hence $U$ and $\pi(x)$ constructed relative to $\Psi$ and $\Psi^{\prime}$ coincide.

Q.E.D.

Corollary. Let $\phi_{0}$ be $*$ automorphism of $\mathfrak{F}$ and $\phi^{\prime}=\phi_{0}^{-1} \phi \phi_{0}$. Then $M\left(\mathfrak{F}, \phi^{\prime}\right)$ is unitarily equivalent to $M(\mathfrak{F}, \phi)$.

Since the triplet $F, \phi, \omega_{T}$ is isomorphic to $\phi_{0}^{-1} \mathfrak{F}=\mathfrak{F}, \phi^{\prime}, \phi_{0}^{*} \omega_{T}$, the triplet $\mathfrak{F}, \phi, \Psi$ is unitarily equivalent to $\mathfrak{F}, \phi^{\prime}, \Psi^{\prime}$ if $\omega_{\Psi^{\prime}}=\phi_{0}^{*} \omega_{\Psi}$ and $\Psi^{\prime}$ is cyclic and separating. Since $\Psi^{\prime}=\alpha \Psi$ for strictly positive selfadjoint $\alpha$ affiliated with $\mathfrak{F}_{c}$ is cyclic and separating, we have Corollary from Theorem 4.

Q.E.D.

It is also obvious that $M(\mathfrak{F}, \phi)$ is unitarily equivalent to $M\left(\mathfrak{F}, \phi \phi_{0}\right)$ for any inner $*$ automorphism $\phi_{0}$. (If $\phi_{0}(x)=u x u^{*}$, then consider $\pi\left(u^{*}\right) U$ instead of $U$.)

Theorem 5. Let $\mathfrak{F}_{1}$ and $\mathfrak{F}_{2}$ be finite von Neumann algebras on $\boldsymbol{\Omega}_{1}$ and $\Re_{2}$ with cyclic and separating unit trace vectors $\Psi_{1}$ and $\Psi_{2}$. Let $\phi_{1}$ and $\phi_{2}$ be injective endomorphisms of $\mathfrak{\mho}_{1}$ and $\mathfrak{\mho}_{2}$ such that $\phi_{k}\left(\mathfrak{F}_{k}\right)=\phi_{k}(1) \mathfrak{\mho}_{k} \phi_{k}(1)$, $\phi_{k}(z)=z \phi_{k}(1)$ for all $z$ in the center of $\widetilde{F}_{k}$ and $\phi_{k}(1)^{\natural}=e^{-a}, k=1,2, a>0$. The pairs $M\left(\mathfrak{\mho}_{1}, \phi_{1}\right), \Omega\left(\mathfrak{F}_{1}, \phi_{1}\right)$ and $M\left(\mathfrak{\mho}_{2}, \phi_{2}\right), \Omega\left(\mathfrak{F}_{2}, \phi_{2}\right)$ are unitarily equivalent if and only if there exists a unitary mapping $w$ from $\Omega_{1}$ onto $\Re_{2}$ and a unitary operator $v$ in $\mathfrak{F}_{2}$ such that $w \mathfrak{F}_{1} w^{*}=\mathfrak{F}_{2}, w \Psi_{1}=\Psi_{2}$ and 
$\phi_{2}^{-1} \phi_{v} \phi_{w} \phi_{1} \phi_{w^{*}}$ is an inner * automorphism of $\mathfrak{F}_{1}$ where $\phi_{u}(x)=u x u^{*}$.

Proof. Let $\bar{w}$ be a unitary mapping such that $\bar{w} \Omega\left(\mathfrak{F}_{1}, \phi_{1}\right)=\Omega\left(\mathfrak{F}_{2}, \phi_{2}\right)$ and $\bar{w} M\left(\mathfrak{F}_{1}, \phi_{1}\right) \bar{w}^{*}=M\left(\mathfrak{F}_{2}, \phi_{2}\right)$. Since $J(\mathfrak{F}, \phi)$ and $\Delta(\mathfrak{F}, \phi)$ are defined by a polar decomposition of the closure $\bar{s}$ of $S$ defined by $S Q \Omega(\mathscr{F}, \phi)=$ $Q^{*} \Omega(\mathfrak{F}, \phi), Q \in M(\mathfrak{F}, \phi)$, we have $\bar{w} \Delta\left(\mathfrak{F}_{1}, \phi_{1}\right) \bar{w}^{*}=\Delta\left(\mathfrak{F}_{2}, \phi_{2}\right)$. Hence $\bar{w} M\left(\mathfrak{\mho}_{1}, \phi_{1}\right)_{0} \bar{w}^{*}=M\left(\mathfrak{\mho}_{2}, \phi_{2}\right)_{0}$. Hence $\bar{w}$ restricted to the eigenspace $\mathfrak{S}_{0}$ of $\Delta\left(\mathfrak{F}_{1}, \phi_{1}\right)$ belonging to an eigenvalue 1 , gives a unitary mapping from the pair $M\left(\mathfrak{F}_{1}, \phi_{1}\right)_{0}, \Omega\left(\mathfrak{F}_{1}, \phi_{1}\right)\left(\sim \mathfrak{F}_{1}, \Psi_{1}\right)$ to $M\left(\mathfrak{F}_{2}, \phi_{2}\right)_{0}, \Omega\left(\mathfrak{F}_{2}, \phi_{2}\right)\left(\sim \mathfrak{F}_{2}, \Psi_{2}\right)$. Let $w$ be the corresponding unitary mapping from $\Re_{1}, \mathfrak{F}_{1}, \Psi_{1}$ to $\Re_{2}, \mathfrak{F}_{2}$, $\Psi_{2}$.

Let $\bar{w} \pi\left(\phi_{1}(1)\right) \bar{w}^{*}=e_{1}, \pi\left(\phi_{2}(1)\right)=e_{2}$. By assumption, $e_{1}^{\natural}=e_{2}^{\natural}=e^{-a}$ where 4 denotes the canonical 4 -mapping in $M\left(\mathfrak{\mho}_{2}, \phi_{2}\right)_{0}$. Hence there exists a unitary $\bar{v} \in M\left(\mathfrak{F}_{2}, \phi_{2}\right)_{0}$ such that $\bar{v} e_{1} \bar{v}^{*}=e_{2}$. Let $\bar{v}=\pi(v), v \in \mathfrak{F}$.

By construction, there exist isometric operators $U_{1}^{*}$ in $M\left(\mathfrak{F}_{1}, \phi_{1}\right)_{-a}$ and $U_{2}^{*}$ in $M\left(\Im_{2}, \phi_{2}\right)_{-a}$ such that $U_{1}^{*} \pi(x) U_{1}=\pi\left(\phi_{1}(x)\right)$ and $U_{2}^{*} \pi(x) U_{2}=$ $\pi\left(\phi_{2}(x)\right)$. Then $\bar{u}=U_{2} \bar{v} \bar{w} U_{1}^{*} \bar{w}^{*}$ is a unitary operator in $M\left(\mathfrak{\mho}_{2}, \phi_{2}\right)_{0}$ and we have $\bar{u} \pi(x) \bar{u}^{*}=\pi\left\{\phi_{2}^{-1} \phi_{v} \phi_{w} \phi_{1} \phi_{w^{*}}(x)\right\}$. Hence $\phi_{2}^{-1} \phi_{v} \phi_{w} \phi_{1} \phi_{w^{*}}$ is inner.

The converse is immediate.

Q.E.D.

Theorem 6. Let $\mathfrak{F}$ be a finite von Neumann algebra with a cyclic and separating trace vector and $\phi_{1}, \phi_{2}$ be two injective endomorphisms of $\mathfrak{F}$ such that $\phi_{k}(\mathfrak{F})=\phi_{k}(1) \mathfrak{F} \phi_{k}(1), \phi_{k}(z)=z \phi_{k}(1)$ for all $z \in \mathfrak{F}_{c}$ and $\phi_{1}(1)^{\natural}=$ $\phi_{2}(1)^{\natural}=e^{-a}, a>0$. Two triplets $\mathfrak{F}, \phi_{1}, \Psi_{1}$ and $\mathfrak{F}, \phi_{2}, \Psi_{2}$ for some cyclic and separating trace vectors $\Psi_{1}$ and $\Psi_{2}$ satisfy the relation in Theorem 5 if and only if there exists $a *$ automorphism $\phi_{0}$ of $\mathfrak{F}$ such that

$$
\phi_{2}^{-1} \phi_{v} \phi_{0}^{-1} \phi_{1} \phi_{0}
$$

is an inner * automorphism of $\mathfrak{F}$ where $v$ is any unitary element in $\mathfrak{F}$ satisfying

$$
v\left[\phi_{0}^{-1} \phi_{1}(1)\right] v^{*}=\phi_{2}(1)
$$

Proof. The "only if" part follows from the condition stated in Theorem 6 because $\phi_{w^{*}}$ is a $*$ isomorphism of $\mathfrak{F}$ if $\widetilde{F}_{1}$ and $\mathfrak{F}_{2}$ are both $*$ isomorphic to $\mathfrak{F}$. The "if" part is also immediate because $x \in \mathfrak{F}$ and its 
cyclic and separating trace vector $\Psi$ is mapped unitarily to $\phi_{0}^{-1}(x) \in \mathfrak{F}$ and $\Psi^{\prime}$ if $\Psi^{\prime}$ is another trace vector such that $\phi_{0}^{*} \omega_{\Psi}=\omega_{\Psi^{\prime}}$.

Q.E.D.

\section{§5. Asymptotic Abelian System}

\section{Proof of Theorem 2}

Notation The Hilbert space, representation of $\mathfrak{M}$ and a cyclic and separating unit vector associated with $\rho$ are denoted by $\mathfrak{E}_{\rho}, \pi_{\rho}$ and $\boldsymbol{\Omega}_{\rho}$. Modular operator and modular conjugation operator for $\Omega_{\rho}$ are denoted by $\Delta_{\rho}$ and $J_{\rho} . \quad \bar{\tau}_{\rho}(t) Q \equiv \Delta_{\rho}^{i t} Q \Delta_{\rho}^{-i t}, j_{\rho}(Q)=J_{\rho} Q J_{\rho}$. $\quad \mathfrak{D}_{a}$ denotes the eigenspace of $\log \Delta_{\rho}$ belonging to an eigenvalue $a$ and $\overline{\mathfrak{M}}_{a}$ is the set of $Q \in \overline{\mathfrak{M}} \equiv \pi(\mathfrak{M})$ satisfying $\bar{\tau}_{\rho}(t) Q=e^{i a t} Q . \quad U_{\alpha}$ is a unitary operator satisfying $U_{\alpha} \pi_{\rho}(Q) \Omega_{\rho}$ $=\pi_{\rho}\left(\tau_{\alpha} Q\right) \Omega_{\rho}$ for all $Q \in \mathfrak{M} . \quad \bar{\tau}_{\alpha}(Q)=U_{\alpha} Q U_{\alpha}^{*}$.

Step 1. $U_{\alpha}$ commutes with $\Delta_{\rho}$.

For $S_{\rho}=J_{\rho} \Delta_{\rho}^{1 / 2}$ and $Q \in \mathfrak{M}$, we have

$$
S_{\rho} U_{\alpha} \pi_{\rho}(Q) \Omega_{\rho}=\pi_{\rho}\left(\tau_{\alpha} Q\right) * \Omega_{\rho}=U_{\alpha} S_{\rho} \pi_{\rho}(Q) \Omega_{\rho},
$$

which implies $\left[U_{\alpha}, S_{\rho}\right]=0$ and hence $\left[U_{\alpha}, \Delta_{\rho}\right]=0$.

Step 2. Let $\bar{s}_{a}$ be the support of $\mathscr{G}_{a}$ in $\overline{\mathfrak{M}}$. Then $\bar{s}_{a} \in \bar{Z}\left(\subset \overline{\mathfrak{B}}_{0}\right)$ and $\bar{s}_{-a}=\bar{s}_{a}$.

Since $\mathfrak{Q}_{a}$ is invariant under $\Delta^{i t}, \bar{s}_{a} \in \overline{\mathfrak{M}}_{0}$. Since $U_{\alpha}$ commute with $\Delta_{\rho}$, $\mathfrak{S}_{a}$ is invariant under $U_{\alpha}$ and hence $\bar{s}_{a}$ commutes with $U_{\alpha}$.

Let $s_{a}=\pi_{\rho}^{-1}\left(\bar{s}_{a}\right)$.

$$
\psi_{a}(Q) \equiv \rho\left(s_{a} Q\right)=\rho\left(s_{a} Q s_{a}\right), \quad Q \in \mathfrak{M}
$$

It is a normal positive linear functional on $\mathfrak{M}$. By using the mapping $F_{\rho}^{3 \mathfrak{M}}$ of $[2]$, we have

$$
\rho\left(s_{a} Q\right)=\rho\left(F_{\rho}^{B M}\left(s_{a}\right) Q\right), \quad Q \in \mathcal{Z} .
$$

By Theorem 1 (6) of [2],

$$
\tau_{\alpha} F_{\rho}^{Z \mathfrak{M}}\left(s_{a}\right)=F_{\rho}^{3 \mathfrak{M}}\left(\tau_{\alpha} s_{a}\right)=F_{\rho}^{3 \mathfrak{M}}\left(s_{a}\right) .
$$

Hence for $Q \in \mathfrak{N}$., we have by Lemma 9 of [3] 


$$
\begin{aligned}
\rho\left(s_{a} Q\right) & =\rho\left(\left(\tau_{\alpha}^{-1} s_{a}\right) Q\right)=\rho\left(s_{a} \tau_{\alpha} Q\right) \\
& =\lim _{\alpha} \rho\left(s_{a} \tau_{\alpha} Q\right)=\lim _{\alpha} \rho\left(F_{\rho}^{3 \mathfrak{M}}\left(s_{a}\right) \tau_{\alpha} Q\right) \\
& =\lim _{\alpha} \rho\left(F_{\rho}^{B \mathfrak{M}}\left(s_{a}\right) Q\right)=\rho\left(F_{\rho}^{3 \mathfrak{M}}\left(s_{a}\right) Q\right) .
\end{aligned}
$$

This implies

$$
\pi_{\rho}\left\{F_{\rho}^{3 \mathfrak{M}}\left(s_{a}\right)-s_{a}\right\} \Omega=0
$$

Since $\rho$ is faithful, we have

$$
s_{a}=F_{\rho}^{3 \mathfrak{M}}\left(s_{a}\right) \in \mathbb{3} \text {. }
$$

By the same argument as Step 3 of the proof of Theorem 1, we have $s_{a}=s_{-a}$.

Step 3. Let $s_{0}(Q)$ denotes the support in $\bar{B}_{0}$ of $Q \in \overline{\mathfrak{M}}$, namely the smallest projection $e \in \overline{\mathfrak{Z}}_{0}$ such that $e Q=Q e=Q$. Then $s_{0}\left(U_{1}^{*} U_{1}\right) \perp$ $s_{0}\left(U_{2}^{*} U_{2}\right)$ is equivalent to $s_{0}\left(U_{1} U_{1}^{*}\right) \perp s_{0}\left(U_{2} U_{2}^{*}\right)$ for $U_{1}, U_{2} \in\left(\overline{\mathfrak{M}}_{a}\right)_{p . i \text {. }}$.

Assume that $s_{0}\left(U_{1}^{*} U_{1}\right) \perp s_{0}\left(U_{2}^{*} U_{2}\right)$ and $s_{0}\left(U_{1} U_{1}^{*}\right) \wedge s_{0}\left(U_{2} U_{2}^{*}\right) \neq 0$. Then there exists $u \in\left(\overline{\mathfrak{M}}_{0}\right)_{p . i \text {. }}$ such that $U_{2}^{*} u U_{1} \neq 0$. However by (3.1) and (3.2), $U_{2}^{*} u U_{1} \in \overline{\mathfrak{M}}_{0}$ and $s_{0}\left(U_{2}^{*} U_{2}\right) U_{2}^{*} u U_{1} s_{0}\left(U_{1}^{*} U_{1}\right)=U_{2}^{*} u U_{1} \neq 0$, which is a contradiction. Hence $s_{0}\left(U_{1}^{*} U_{1}\right) \perp s_{0}\left(U_{2}^{*} U_{2}\right)$ implies $s_{0}\left(U_{1} U_{1}^{*}\right) \perp s_{0}\left(U_{2} U_{2}^{*}\right)$. Similarly the converse is proved.

Step 4. $s_{0}\left(U_{1}^{*} U_{1}\right)=s_{0}\left(U_{2}^{*} U_{2}\right)$ is equivalent to $s_{0}\left(U_{1} U_{1}^{*}\right)=s_{0}\left(U_{2} U_{2}^{*}\right)$ for $U_{1}, U_{2} \in\left(\overline{\mathfrak{M}}_{a}\right)_{p . i .}$.

Assume that a projection $e \in \overline{\mathbf{Z}}_{0}$ satisfies

$$
s_{0}\left(U_{1}^{*} U_{1}\right) \perp e, s_{0}\left(U_{2}^{*} U_{2}\right) \geqq e \neq 0 .
$$

Then $u_{2}=U_{2} e \in\left(\overline{\mathfrak{M}}_{a}\right)_{p . i .}, \quad u_{2}^{*} u_{2}=e U_{2}^{*} U_{2} e \neq 0, s_{0}\left(u_{2}^{*} u_{2}\right)=e . \quad$ By Step 3 , $s_{0}\left(u_{2} u_{2}^{*}\right) \perp s_{0}\left(U_{1} U_{1}^{*}\right)$. We also have $s_{0}\left(u_{2} u_{2}^{*}\right)=s_{0}\left(U_{2} e U_{2}^{*}\right) \leqq s_{0}\left(U_{2} U_{2}^{*}\right)$. Similarly, $e \in \overline{\mathbf{Z}}_{0}, s_{0}\left(U_{2}^{*} U_{2}\right) \perp e$ and $s_{0}\left(U_{1}^{*} U_{1}\right) \geqq e \neq 0$ imply $s_{0}\left(U_{2} U_{2}^{*}\right) \perp$ $s_{0}\left(u_{1} u_{1}^{*}\right)$ and $s_{0}\left(U_{1} U_{1}^{*}\right) \geqq s_{0}\left(u_{1} u_{1}^{*}\right) \neq 0$ for $u_{1}=U_{1} e$. Hence $s_{0}\left(U_{1}^{*} U_{1}\right) \neq$ $s_{0}\left(U_{2}^{*} U_{2}\right)$ implies $s_{0}\left(U_{1} U_{1}^{*}\right) \neq s_{0}\left(U_{2} U_{2}^{*}\right)$.

The converse is similarly proved. 


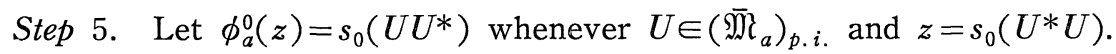
Then $\phi_{a}^{0}$ is a lattice automorphism of projections in $\bar{s}_{a} \overline{3}_{0}$.

By Step $4, \phi_{a}^{0}$ is single-valued. By Step 3, $z_{1} \perp z_{2}$ and $\phi_{a}^{0}\left(z_{1}\right) \perp \phi_{a}^{0}\left(z_{2}\right)$ are equivalent,

If $U \in\left(\overline{\mathfrak{M}}_{a}\right)_{p . i .}$, then $U \Omega_{\rho}=s_{a} U \Omega_{\rho}$ and hence $U U^{*} \leqq \bar{s}_{a}$. We also have $U^{*} \in\left(\overline{\mathfrak{M}}_{-a}\right)_{p . i .}$ and hence $U^{*} U \leqq \bar{s}_{-a}=\bar{s}_{a}$.

Let $U_{\nu}$ be a maximal family of elements in $\left(\overline{\mathfrak{M}}_{a}\right)_{p . i}$. such that $s_{0}\left(U_{\nu}^{*} U_{\nu}\right)$ is mutually orthogonal. Then $s_{0}\left(U_{\nu} U_{\nu}^{*}\right)$ is mutually orthogonal by Step 3 and hence $U=\sum_{\nu} U_{\nu}$ is in $\left(\overline{\mathfrak{M}}_{a}\right)_{p . i .}$. If $s_{0}\left(U^{*} U\right)<\bar{s}_{a}$, then there exists non-zero $u \in\left(\overline{\mathfrak{M}}_{a}\right)_{p . i}$. with $s_{0}\left(u^{*} u\right) \perp s_{0}\left(U^{*} U\right)$, by Step 3 of Section 3 . By Step 3, $s_{0}\left(u u^{*}\right) \perp s_{0}\left(U U^{*}\right)$. This contradicts the maximality. Hence $s_{0}\left(U^{*} U\right)=\bar{s}_{a}$. Similarly $s_{0}\left(U U^{*}\right)=\bar{s}_{a}$ by Step 2 of Section 3, Step 3 and maximality.

For any projection $e \in \overline{\mathfrak{Z}}_{0} \bar{s}_{a}, U e \in\left(\overline{\mathfrak{M}}_{a}\right)_{p . i .}, e U \in\left(\overline{\mathfrak{M}}_{a}\right)_{p . i .}, s_{0}\left((U e)^{*}(U e)\right)=$ $e s_{0}\left(U^{*} U\right) e=e$ and $s_{0}\left((e U)(e U)^{*}\right)=e s_{0}\left(U U^{*}\right) e=e$. Hence the domain and range of $\phi_{a}^{0}$ is all projections in $\bar{s}_{a} \overline{\mathrm{B}}_{0}$.

Since $z_{1} \geqq z_{2}$ is equivalent to $z_{2} \perp z$ for all $z \perp z_{1}, z_{1} \geqq z_{2}$ and $\phi_{a}^{0}\left(z_{1}\right)$ $\geqq \phi_{a}^{0}\left(z_{2}\right)$ are equivalent. Hence $\phi_{a}^{0}$ is a lattice automorphism.

Step 6. If $z=\int \lambda d e_{\lambda}+i \int \lambda d e_{\lambda}^{\prime} \in \overline{\mathrm{Z}}_{0} \bar{s}_{a}$, then define $\phi_{a}^{0}(z)=\int \lambda d \phi_{a}^{0}\left(e_{\lambda}\right)+$ $i \int \lambda d \phi_{a}^{0}\left(e_{\lambda}^{\prime}\right)$. Then $\phi_{a}^{0}$ is an automorphism of $\overline{3}_{0} \bar{s}_{a}$, which follows from Step 5 .

Step 7. If $z \in \overline{\mathfrak{B}}_{0} \bar{s}_{a}$ and $U \in\left(\overline{\mathfrak{M}}_{a}\right)_{p . i .}$, then $\phi_{a}^{0}(z) U=U z$.

It is enough to prove the equation for a projection $z \in \overline{\mathbf{Z}}_{0} \bar{s}_{a}$. We have

$$
s_{0}((U z) *(U z))=z s_{0}\left(U^{*} U\right) z=s_{0}\left(U^{*} U\right) \wedge z .
$$

Since $\phi_{a}^{0}$ is a lattice automorphism, we have

$$
s_{0}\left((U z)(U z)^{*}\right)=s_{0}\left(U U^{*}\right) \wedge \phi_{a}^{0}(z) .
$$

Since $s_{0}\left(U U^{*}\right) U=U$, we have

$$
U z=s_{0}\left\{(U z)(U z)^{*}\right\} U z=\phi_{a}^{0}(z) U z
$$

Similarly we have 


$$
\begin{aligned}
\phi_{a}^{0}(z) U & =\phi_{a}^{0}(z) U s_{0}\left\{\left(\phi_{a}^{0}(z) U\right)^{*}\left(\phi_{a}^{0}(z) U\right)\right\} \\
& =\phi_{a}^{0}(z) U\left\{\left(\phi_{a}^{0}\right)^{-1} \phi_{a}^{0}(z) \wedge s_{0}\left(U^{*} U\right)\right\} \\
& =\phi_{a}^{0}(z) U z=U z .
\end{aligned}
$$

Step 8. $\phi_{a}^{0}(z)=z$ if $z \in \overline{3}_{a}$.

It is enough to prove it for a projection $z$.

Let $z \in \bar{乃}_{a}, s_{0}\left(U^{*} U\right)=z$. Then $U z=U=z U$. Hence $s_{0}\left(U U^{*}\right) \leqq z$. Let $z-s_{0}\left(U U^{*}\right) \equiv e,\left(\phi_{a}^{0}\right)^{-1} e \equiv e^{\prime}$. Let $u \in\left(\overline{\mathfrak{M}}_{a}\right)_{p . i .}, u^{*} u=e^{\prime}, u u^{*}=e$. Since $z \geqq e$ and $z \in \mathcal{Z}, u=z u=u z$. Hence $e^{\prime} \leqq z$. On the other hand $e \perp \phi_{a}^{0}(z)$ implies $e^{\prime} \perp z$. Hence $e^{\prime}=0$. Hence $z=s_{0}\left(U U^{*}\right)=\phi_{a}^{0}(z)$.

Step 9. $\bar{\tau}_{\alpha}$ leaves $\overline{3}_{0} \bar{s}_{a}$ invariant and commutes with $\phi_{a}^{0}$.

Since $U_{\alpha}$ commutes with $\Delta_{\rho}, \bar{B}_{0}$ remains invariant (as a set) under $\bar{\tau}_{\alpha} . \bar{s}_{a}$ commutes with $U_{\alpha}$ as we have seen in Step 2. $\bar{\tau}_{\alpha}$ also leaves $\left(\overline{\mathfrak{M}}_{a}\right)_{p . i .}$ invariant as a set. We now have

$$
\begin{aligned}
& \bar{\tau}_{\alpha} s_{0}\left(U^{*} U\right)=s_{0}\left(\left(\bar{\tau}_{\alpha} U\right)^{*}\left(\bar{\tau}_{\alpha} U\right)\right) \\
& \bar{\tau}_{\alpha} s_{0}\left(U U^{*}\right)=s_{0}\left(\left(\bar{\tau}_{\alpha} U\right)\left(\bar{\tau}_{\alpha} U\right)^{*}\right)
\end{aligned}
$$

because $\mathfrak{M}_{0}$ is also invariant under $\bar{\tau}_{\alpha}$ as a set. This implies $\bar{\tau}_{\alpha} \phi_{a}^{0}=\phi_{a}^{0} \bar{\tau}_{\alpha}$.

Step 10. $\left(\Omega_{\rho}, \phi_{a}^{0}(z) \Omega_{\rho}\right)=\left(\Omega_{\rho}, z \Omega_{\rho}\right)$ for $z \in \overline{\mathbf{Z}}_{0} \bar{s}_{a}$.

By Radon-Nikodym theorem, there exists a strictly positive selfadjoint operator $A_{a}^{0}$ affiliated with $\bar{B}_{0} \bar{s}_{a}$ such that

$$
\left(\Omega_{\rho}, \phi_{a}^{0}(z) \Omega_{\rho}\right)=\left(A_{a}^{0} \Omega_{\rho}, z A_{a}^{0} \Omega\right)
$$

Since $\bar{\tau}_{\alpha}$ commutes with $\phi_{a}^{0}$ and $\rho$ is invariant under $\bar{\tau}_{\alpha}, A_{a}^{0}$ must be invariant under $\bar{\tau}_{\alpha}$, namely $A_{a}^{0}=\int \lambda d e_{\lambda},\left[e_{\lambda}, U_{\alpha}\right]=0$. By the same argument as for $\bar{s}_{a}$ in Step 2, we obtain $e_{\lambda} \in \overline{3}$. Namely $\left(A_{a}^{0}\right)^{2}$ is the RadonNikodym derivative of $\left(\phi_{a}^{0}\right) * \bar{\rho}$ by $\bar{\rho}$ relative to $\overline{3}$ where $\bar{\rho}=\rho \circ \pi^{-1}$. Since $\phi_{a}^{0}=1$ on $\overline{3} \bar{s}_{a}$, we have $A_{a}^{0}=1$.

Step 11. $\left(U U^{*}\right)^{\natural}=e^{a} \phi_{a}^{0}\left\{\left(U^{*} U\right)^{\natural}\right\}$ for $U \in\left(\overline{\mathfrak{M}}_{a}\right)_{p . i,}$. Let $z \in \overline{3}_{0} \bar{s}_{a}$. Then 


$$
\begin{array}{rlrl}
\left(\Omega_{\rho}, U^{*} U z \Omega_{\rho}\right) & =\left(\Omega_{\rho}, U^{*} \phi_{a}^{0}(z) U \Omega_{\rho}\right) & & \text { (by Step 7) } \\
& =\left(\Omega_{\rho}, U^{*} \phi_{a}^{0}(z) j_{\rho}\left(U^{*}\right) \Omega_{\rho}\right) e^{-a / 2} & & \left(\text { by } U^{*} \Omega_{\rho} \in \mathfrak{S}_{-a}\right) \\
& =\left(j_{\rho}(U) \Omega_{\rho}, U^{*} \phi_{a}^{0}(z) \Omega_{\rho}\right) e^{-a / 2} & \\
& =\left(\Omega_{\rho}, U U^{*} \phi_{a}^{0}(z) \Omega_{\rho}\right) e^{-a} & \\
& =\left(\Omega_{\rho},\left(U U^{*}\right)^{\natural} \phi_{a}^{0}(z) \Omega_{\rho}\right) e^{-a} & & \text { (by Lemma 7) } \\
& =\left(\Omega_{\rho},\left\{\left(\phi_{a}^{0}\right)^{-1}\left(U U^{*}\right)^{\natural}\right\} z \Omega_{\rho}\right) e^{-a} & & \text { (by Step 10). }
\end{array}
$$

Hence

$$
\left(U^{*} U\right)^{\natural}=e^{-a}\left(\phi_{a}^{0}\right)^{-1}\left\{\left(U U^{*}\right)^{\natural}\right\} .
$$

Step 12. There exists $U_{a} \in\left(\mathfrak{M}_{a}\right)_{p . i}$. such that $U_{a} U_{a}^{*}=s_{a}$ if $a>0$.

The proof is the same as Step 5 of Section 3, except the inequality is now proved using Step 11 as follows:

$$
\begin{aligned}
\left(s_{a}-U_{0}^{*} U_{0}\right)^{\natural} & =s_{a}-e^{-a}\left(\phi_{a}^{0}\right)^{-1}\left\{\left(U U^{*}\right)^{\natural}\right\} \\
& \geqq e^{-a}\left(\phi_{a}^{0}\right)^{-1}\left\{\left(s_{a}-U U^{*}\right)^{\natural}\right\} \\
& \geqq e^{-a}\left(\phi_{a}^{0}\right)^{-1}\left\{\left(U^{\prime} U^{\prime *}\right)^{\natural}\right\}=\left(U^{\prime} * U^{\prime}\right)^{\natural} .
\end{aligned}
$$

Step 13. There exists $N_{a} \in\left(\overline{\mathfrak{M}}_{a}\right)_{p . i .}$ such that $N_{a} N_{a}^{*}+N_{a}^{*} N_{a}=\bar{s}_{a}$, $\left(N_{a}^{*} N_{a}\right)^{\natural}=\left(1+e^{a}\right)^{-1} \bar{s}_{a}$.

For any $T \in \bar{s}_{a} \overline{\mathrm{Z}}_{0}, 0 \leqq T \leqq 1$, there exists a projection $e_{T} \in\left(\overline{\mathfrak{M}}_{0} \bar{s}_{a}\right)$ such that $\left(e_{T}\right)^{\natural}=T$. Let $e_{(0)}$ be a projection in $\left(\overline{\mathfrak{M}}_{0} \bar{s}_{a}\right)$ such that $\left(e_{(0)}\right)^{\natural}=$ $\left(1+e^{-a}\right)^{-1} \bar{s}_{a}$. Let $U_{(0)}=e_{(0)} U_{a}$. Then $U_{(0)} U_{(0)}^{*}=e_{(0)}$ and hence

$$
\left(U_{(0)}^{*} U_{(0)}\right)^{\natural}=e^{-a}\left(\phi_{a}^{0}\right)^{-1}\left\{e_{0}^{\natural}\right\}=\left(1+e^{a}\right)^{-1} \bar{s}_{a}=\left(\bar{s}_{a}-e_{(0)}\right)^{\natural} .
$$

There exists $u \in\left(\overline{\mathfrak{M}}_{0} \bar{s}_{a}\right)_{p . i}$. such that

$$
u u^{*}=U_{(0)}^{*} U_{(0)}, u^{*} u=\bar{s}_{a}-e_{(0)}
$$

Setting $U_{(0)} u=N_{a}$, we have $N_{a} \in\left(\overline{\mathfrak{M}}_{a}\right)_{p . i .}$,

$$
N_{a}^{*} N_{a}=u^{*} u=\bar{s}_{a}-e_{(0)}, \quad N_{a} N_{a}^{*}=U_{(0)} U_{(0)}^{*}=e_{(0)} .
$$

Hence $N_{a}^{*} N_{a}+N_{a} N_{a}^{*}=\bar{s}_{a}$. We have $\left(N_{a}^{*} N_{a}\right)^{\natural}=\bar{s}_{a}-\left(e_{(0)}\right)^{\natural}=\left(1+e^{a}\right)^{-1} \bar{s}_{a}$. 
Step 14. $z \in \overline{\mathfrak{B}}_{0} \bar{s}_{a}$ commutes with $\pi_{\rho}(\mathfrak{V})_{a} \equiv \overline{\mathfrak{M}}_{a} \cap \pi_{\rho}(\mathfrak{X})$.

Let $Q, Q^{\prime} \in \pi_{\rho}(\mathfrak{2})_{a}$.

Then $Q \bar{\tau}_{\alpha}^{-1}\left(N_{a}^{*}\right) \in \overline{\mathfrak{M}}_{0}$, it commutes with $z$. We have

$$
\begin{aligned}
& \left(Q^{\prime} \Omega_{\rho}, Q \bar{\tau}_{a}^{-1}\left(N_{a}^{*}\right) z \bar{\tau}_{\alpha}^{-1}\left(N_{a}\right) \Omega_{\rho}\right) \\
& \quad=\left(Q^{\prime} \Omega_{\rho}, z Q \bar{\tau}_{\alpha}^{-1}\left(N_{a}^{*} N_{a}\right) \Omega_{\rho}\right) .
\end{aligned}
$$

The functional $f(Q)=\left(\Omega_{\rho}, Q N_{a}^{*} N_{a} \Omega_{\rho}\right)$ satisfies

$$
\begin{aligned}
f(Q) & =\left(\Omega_{\rho}, Q N_{a}^{*} J_{\rho} \Delta_{\rho}^{1 / 2} N_{a}^{*} \Omega\right) \\
& =\left(\Omega_{\rho}, Q N_{a}^{*} j_{\rho}\left(N_{a}^{*}\right) \Omega_{\rho}\right) e^{-a / 2} \\
& =\left(j_{\rho}\left(N_{a}\right) \Omega_{\rho}, Q N_{a}^{*} \Omega_{\rho}\right) e^{-a / 2} \\
& =\left(N_{a}^{*} \Omega_{\rho}, Q N_{a}^{*} \Omega_{\rho}\right) e^{-a} \geqq 0
\end{aligned}
$$

for $Q \in \overline{\mathfrak{M}}, Q \geqq 0$ and

$$
f(w)=\left(\Omega_{\rho}, w\left(N_{a}^{*} N_{a}\right)^{\natural} \Omega_{\rho}\right)=\left(1+e^{a}\right)^{-1}\left(\Omega_{\rho}, \bar{s}_{a} w \Omega_{\rho}\right)
$$

for $w \in 3_{0}$.

Since the functional $\left(\Omega_{\rho}, \bar{s}_{a} Q \Omega_{\rho}\right)=\left(\Omega_{\rho}, \bar{s}_{a} Q \bar{s}_{a} \Omega_{\rho}\right)$ is invariant under $\bar{\tau}_{\alpha}$ and since $Q^{\prime *} z Q \bar{s}_{a}$ is weakly $\tau_{\alpha}$-central in $\overline{\mathfrak{M}}_{0}$, we have by Lemma 9 of [3],

$$
\begin{aligned}
\left(Q^{\prime} \Omega_{\rho},\right. & \left.z Q \bar{\tau}_{\alpha}^{-1}\left(N_{a}^{*} N_{a}\right) \Omega_{\rho}\right) \\
= & \left(\Omega_{\rho}, \bar{\tau}_{\alpha}\left\{Q^{\prime *} z Q \bar{s}_{a}\right\}\left(N_{a}^{*} N_{a}\right) \Omega_{\rho}\right) \\
& \rightarrow\left(1+e^{a}\right)^{-1}\left(\Omega_{\rho}, Q^{*} z Q \bar{s}_{a} \Omega_{\rho}\right) .
\end{aligned}
$$

On the other hand, we have $Q^{*} Q^{\prime} \Omega_{\rho}=j_{\rho}\left(Q^{*} Q^{\prime}\right)^{*} \Omega_{\rho}$ by $Q^{*} Q^{\prime} \in \overline{\mathfrak{M}}_{0}$. Hence

$$
\left(Q^{\prime} \Omega_{\rho}, Q \bar{\tau}_{a}^{-1}\left(N_{a}^{*}\right) z \bar{\tau}_{a}^{-1}\left(N_{a}\right) \Omega_{\rho}\right)=\left(N_{a} \Omega_{\rho}, \bar{\tau}_{\alpha}\left(j_{\rho}\left(Q^{*} Q^{\prime}\right) z\right) N_{a} \Omega_{\rho}\right)
$$

The $*$ subalgebra generated by $\pi_{\rho}\left(\mathfrak{U}_{0}\right)_{0}$ and $\bar{Z}_{0}$ is strongly $\bar{\tau}_{\alpha}$ central in $\overline{\mathfrak{M}}_{0}$. We now prove that this algebra is strongly dense in $\overline{\mathfrak{M}}_{0}$. Let $Q \in \overline{\mathfrak{M}}_{0}, \Phi_{i} \in \mathfrak{S}, i=1, \ldots, n$ and $\varepsilon>0$. Let $\tilde{f}$ be a $C^{\infty}$ function such that its 
support is in $\left[-a_{1} / 2, a_{1} / 2\right]$ and $\tilde{f}(0)=1$. Let $f(\lambda)=(2 \pi)^{-1} \int \tilde{f}(p) e^{-i p \lambda} d p$, $Q(f)=\int(\bar{\tau}(t) Q) f(t) d t$ and $c=\int|f(\lambda)| d \lambda(\geqq \tilde{f}(0)=1)$. Since $\Omega_{p}$ is separating for $\overline{\mathfrak{M}}_{0}$, there exists $Q_{i} \in \overline{\mathfrak{M}}_{0}^{\prime}$ such that $\left\|\Phi_{i}-Q_{i} \Omega_{\rho}\right\|<(4\|Q\| c)^{-1} \varepsilon$. Since $\mathfrak{A}$ is weakly dense in $\mathfrak{M}$, the unit ball of $\pi_{\rho}(\mathfrak{Q})$ is strongly dense in the unit ball of $\overline{\mathfrak{M}}$ and there exists $Q_{\varepsilon} \in \pi_{\rho}(\mathfrak{R})$ satisfying $\left\|Q_{\varepsilon}\right\| \leqq\|Q\|$ and $\|\left(Q_{\varepsilon}\right.$ $-Q) \Omega_{\rho} \|<\left(2 c \max \left\|Q_{i}\right\|\right)^{-1} \varepsilon$. Then $\left\|\left\{Q_{\varepsilon}(f)-Q(f)\right\} \Omega_{\rho}\right\|<\left(2 \max \left\|Q_{i}\right\|\right)^{-1} \varepsilon$. Since $Q \in \overline{\mathfrak{M}}_{0}$, we have $Q(f)=Q$. Due to the assumed support of $\tilde{f}$, $Q_{\varepsilon}(f) \Omega_{\rho} \in \mathfrak{S}_{0}$ and hence $\left\{\bar{\tau}(t) Q_{\varepsilon}(f)\right\} \Omega_{\rho}=Q_{\varepsilon}(f) \Omega_{\rho}$. Hence $\left.Q_{\varepsilon}(f) \in \pi_{\rho}(\mathfrak{Q})\right)_{0}$. We have

$$
\begin{aligned}
&\left\|\left(Q_{\varepsilon}(f)-Q(f)\right) \Phi_{i}\right\| \leqq\left(\left\|Q_{\varepsilon}(f)\right\|+\|Q\|\right)\left\|\Phi_{i}-Q_{i} \Omega_{\rho}\right\| \\
&+\left\|Q_{i}\right\|\left\|\left(Q_{\varepsilon}(f)-Q(f)\right) \Omega_{\rho}\right\|<\varepsilon
\end{aligned}
$$

Hence $\pi_{\rho}(\mathfrak{U})_{0}$ is dense in $\overline{\mathfrak{M}}_{0}$.

We can now use Lemma 1 of [3] to obtain

$$
\begin{aligned}
& \left(N_{a} \Omega_{\rho}, \bar{\tau}_{\alpha}\left(j_{\rho}\left(Q^{*} Q^{\prime}\right) z\right) N_{a} \Omega_{\rho}\right) \\
\rightarrow & \left(1+e^{a}\right)^{-1}\left(\Omega_{\rho}, \bar{s}_{a} j_{\rho}\left(Q^{*} Q^{\prime}\right) z \Omega_{\rho}\right) \\
= & \left(Q^{\prime} \Omega_{\rho}, Q z \bar{s}_{a} \Omega_{\rho}\right)\left(1+e^{a}\right)^{-1} .
\end{aligned}
$$

We now have

$$
\left(Q^{\prime} \Omega_{\rho},[z, Q] \bar{s}_{a} \Omega_{\rho}\right)=0
$$

By Lemma 5 of $[3], Q^{\prime} \Omega_{\rho}$ is dense in $\mathfrak{L}_{a}$. Since $[z, Q] \bar{s}_{a} \Omega_{\rho} \in \mathfrak{L}_{a}$, we have

$$
[z, Q] \bar{s}_{a} \Omega_{\rho}=0
$$

Since $\bar{s}_{a} \in Z,[z, Q] \bar{s}_{a}=\left[z \bar{s}_{a}, Q\right]=[z, Q]$ by assumption $z \in \mathcal{Z}_{0} \bar{s}_{a}$. Since $\Omega_{\rho}$ is separating $[z, Q]=0$.

Step 15. $\overline{3}_{0}=\overline{3}$.

By the same proof as that of Lemma $5, \cup_{a} \pi_{\rho}(\mathfrak{H})_{a}$ is total in $\overline{\mathfrak{M}}$. Since $Q \Omega_{\rho} \in \mathfrak{K}_{a}$ for $Q \in \overline{\mathfrak{M}}_{a},\left(1-\bar{s}_{a}\right) Q \Omega_{\rho}=0$ and hence $\left(1-\bar{s}_{a}\right) Q=0$ for $Q \in \pi_{\rho}(\mathfrak{U})_{a} \subset \overline{\mathfrak{M}}_{a}$. Hence $Q \in \pi_{\rho}(\mathfrak{U})_{a}$ commutes with $\left(1-\bar{s}_{a}\right) z$ trivially and 
with $\bar{s}_{a} z$ by Step 14 if $z \in \overline{\mathfrak{Z}}_{0}$. Hence $z \in \overline{\mathrm{Z}}_{0}$ commutes with $\pi_{\rho}(\mathfrak{V})_{a}$ for any $a$ and hence belongs to $\overline{3}$.

Step 16. $r_{\infty}\left(P_{n} M\right)=S_{x_{n}}$.

Since the spectrum of modular operator for $P_{n} \Omega$ is $S_{x_{n}}, x_{n}=e^{-a_{n}}=$ $\phi_{n}(1)^{\natural}$, we have by $[6]$

$$
r_{\infty}\left(P_{n} M\right) \subset S_{x_{n}}
$$

We now show that $P_{n} M$ has the property $L_{\lambda_{n}}^{\prime}$ with $\lambda_{n}=\left(1+x_{n}\right)^{-1} x_{n}$ which shows

$$
r_{\infty}\left(P_{n} M\right)=S_{x_{n}}
$$

by $[1]$, if the space is separable.

The same computation as (5.1) shows

$$
\left(\Omega_{\rho}, Q N_{a} \Omega_{\rho}\right)=\left(\Omega_{\rho}, N_{a} Q \Omega_{\rho}\right) e^{-a}
$$

for all $Q \in \overline{\mathfrak{M}}$. Since $\rho$ is $\tau_{\alpha}$ invariant, we have

$$
\begin{aligned}
\left(\Omega_{\rho}, Q \bar{\tau}_{\alpha}^{-1}\left(N_{a}\right) \Omega_{\rho}\right) & =\left(\Omega_{\rho}, \bar{\tau}_{\alpha}(Q) N_{a} \Omega_{\rho}\right) \\
& =e^{-a}\left(\Omega_{\rho}, N_{a} \bar{\tau}_{\alpha}(Q) \Omega_{\rho}\right)=e^{-a}\left(\Omega_{\rho}, \bar{\tau}_{\alpha}^{-1}\left(N_{a}\right) Q \Omega_{\rho}\right)
\end{aligned}
$$

Therefore

$$
\left(1-\lambda_{n}\right)\left(\Omega_{\rho}, Q \bar{\tau}_{\alpha}^{-1}\left(N_{a_{n}}\right) \Omega_{\rho}\right)=\lambda_{n}\left(\Omega_{\rho}, \bar{\tau}_{\alpha}^{-1}\left(N_{a_{n}}\right) Q \Omega_{\rho}\right)
$$

For any $Q \in \pi_{\rho}(\mathfrak{A})$, let

$$
Q\left(g_{n}\right)=\int \bar{\tau}_{\rho}(t) Q g_{n}(t) d t
$$

where $g_{n}$ is given in the proof of Lemma 5 . It belongs to $\pi_{\rho}(\mathfrak{U})$ and $\lim _{n \rightarrow \infty} Q\left(g_{n}\right)=Q$. Furthermore

$$
\bar{\tau}_{\rho}(z) Q\left(g_{n}\right)=\int \bar{\tau}_{\rho}(t) Q g(n(t-z)) n d t
$$

for real $z$ and the right hand side has an analytic continuation to all complex $z$ as $\pi_{\rho}(\mathfrak{2})$-valued function. We have 


$$
Q\left(g_{n}\right) \Omega_{\rho}=J_{\rho} \Delta_{\rho}^{1 / 2} Q\left(g_{n}\right) * \Omega_{\rho}=j_{\rho}\left(\bar{\tau}_{\rho}(i / 2) Q\left(g_{n}\right)\right) * \Omega_{\rho}
$$

which can be proven by analytic continuation.

Let $\Psi_{j}, j=1, \ldots, k$ be a finite collection of vectors in $\mathfrak{S}_{\rho}$. Then there exist $Q_{j} \in \pi_{\rho}(A)$ and $n$ for any given $\varepsilon>0$, such that $Q_{j}^{\prime}=Q_{j}\left(g_{n}\right)$ satisfies

$$
\left\|\Psi_{j}\right\|\left\|\Psi_{j}-Q_{j}^{\prime} \Omega_{\rho}\right\|<\varepsilon / 4,\left\|Q_{j}^{\prime} \Omega_{\rho}\right\| \leqq\left\|\Psi_{j}\right\|
$$

Then

$$
\begin{gathered}
\left\|\left[N_{a}, \bar{\tau}_{\alpha}\left(Q_{j}^{\prime}\right)\right] \Omega_{\rho}\right\|^{2}=\left(\Omega_{\rho}, N_{a}^{*} \bar{\tau}_{\alpha}(X) N_{a} \Omega_{\rho}\right), \\
X \equiv Q_{j}^{\prime *} Q_{j}^{\prime}-Q_{j}^{\prime *} j_{\rho}\left(\bar{\tau}_{\rho}(i / 2) Q_{j}^{\prime}\right)^{*}-j_{\rho}\left(\bar{\tau}_{\rho}(i / 2) Q_{j}^{\prime}\right) Q_{j}^{\prime} \\
\quad+j_{\rho}\left(\bar{\tau}_{\rho}(i / 2) Q_{j}^{\prime}\right) j_{\rho}\left(\bar{\tau}_{\rho}(i / 2) Q_{j}^{\prime}\right)^{*} .
\end{gathered}
$$

If $z \in \bar{\Omega}$, then

$$
\begin{aligned}
\omega_{N_{a} \Omega_{\rho}}(z) & =\left(\Omega_{\rho}, N_{a}^{*} N_{a} z \Omega_{\rho}\right) \\
& =e^{-a}\left(\Omega_{\rho}, N_{a} N_{a}^{*} z \Omega_{\rho}\right) \quad(\text { by }(5.2)) \\
& =\left(1+e^{a}\right)^{-1}\left(\Omega_{\rho}, \bar{s}_{a} z \Omega_{\rho}\right) \\
& =\left(1+e^{a}\right)^{-1} \omega_{\bar{s}_{a} \Omega_{\rho}}(z) .
\end{aligned}
$$

Since $\omega_{\bar{s}_{a} \Omega_{p}}$ is $\bar{\tau}_{\alpha}$ invariant, we have by Lemma 1 of $[3]$,

$$
\lim _{\alpha} \omega_{N_{a} \Omega_{\rho}}\left(\bar{\tau}_{\alpha}(X)\right)=\left(1+e^{a}\right)^{-1}\left(\Omega_{\rho}, \bar{s}_{a} X \Omega_{\rho}\right)=0 .
$$

Hence there exists $\alpha$ such that $N_{\varepsilon}^{\prime} \equiv \bar{\tau}_{\alpha}^{-1}\left(N_{a_{n}}\right)$ satisfies

$$
\begin{aligned}
& \left\|\Psi_{j}\right\|\left\|N_{\varepsilon}^{\prime} Q_{j}^{\prime} \Omega_{\rho}-Q_{j}^{\prime} N_{\varepsilon}^{\prime} \Omega_{\rho}\right\| \\
& =\left\|\Psi_{j}\right\|\left\|\left[N_{a_{n}}, \tau_{\alpha}\left(Q_{j}^{\prime}\right)\right] \Omega_{\rho}\right\|<\varepsilon / 2, \\
& \left\|\Psi_{j}\right\|\left\|N_{\varepsilon}^{\prime *} Q_{j}^{\prime} \Omega_{\rho}-Q_{j}^{\prime} N_{j}^{\prime *} \Omega\right\| \\
& =\left\|\Psi_{j}\right\|\left\|\left[N_{a_{n}}^{*}, \tau_{\alpha}\left(Q_{j}^{\prime}\right)\right] \Omega_{\rho}\right\|<\varepsilon / 2 .
\end{aligned}
$$

where the second inequality is obtained in a similar manner as the first.

Let $\omega_{1} \cdots \omega_{k}$ be normal states of $P_{n} \mathfrak{M}$. Since $\pi_{\rho}\left(P_{n}\right) \Omega_{\rho}$ is cyclic and separating for $P_{n} \mathfrak{M}$ on $\pi_{\rho}\left(P_{n}\right) \mathfrak{L}_{\rho}$, there exists $\Psi_{j} \in \pi_{\rho}\left(P_{n}\right) \mathfrak{S}_{\rho}$ such that 
$\omega_{\Psi j} \circ \pi_{\rho}=\omega_{j} . \quad$ For $Q \in P_{n} \mathfrak{M}$ and $N_{\varepsilon}=P_{n} \pi_{\rho}^{-1} N_{\varepsilon}^{\prime}$, we have from (5.2) (5.5)

$$
\begin{aligned}
& \left|\lambda_{n} \omega_{j}\left(N_{\varepsilon} Q\right)-\left(1-\lambda_{n}\right) \omega_{j}\left(Q N_{\varepsilon}\right)\right| \\
& \quad=\left|\lambda_{n} \omega_{\Psi^{j}}\left(N_{\varepsilon}^{\prime} \pi_{\rho}(Q)\right)-\left(1-\lambda_{n}\right) \omega_{\Psi_{j}}\left(\pi_{\rho}(Q) N_{\varepsilon}^{\prime}\right)\right| \leqq \varepsilon\|Q\|,
\end{aligned}
$$

$j=1, \ldots, k . \quad N_{\varepsilon}$ is a partial isometry in $P_{n} \mathfrak{M}$ satisfying

$$
N_{\varepsilon}^{*} N_{\varepsilon}+N_{\varepsilon} N_{\varepsilon}^{*}=P_{n}
$$

Hence it also satisfies $N_{\varepsilon}^{2}=0$. This proves the property $L_{\lambda_{n}}^{\prime}$.

Step 17. $r_{\infty}\left(P_{0} \mathfrak{M}\right)=r_{\infty}\left(P \mathfrak{M}_{0}\right)=\{1\}$.

By the proof of Step 16 applied to $a=0, \mathfrak{M \varepsilon _ { 0 }}$ satisfies the property $L_{1 / 2}^{\prime}$. Hence $P M_{0}$ with any central projection $P$ satisfies the same property. Since $r_{\infty}(\Re) \subset\{1\}$ for any finite von Neumann algebra $\Re[4]$, we have $r_{\infty}\left(P_{0} \mathfrak{M}\right)=r_{\infty}\left(P_{n} \mathfrak{M l}_{0}\right)=\{1\}$ if the space is separable by $[1]$.

Q.E.D.

\section{§6. Discussions}

For a von Neumann algebra $\mathfrak{M}$, the relation $r_{\infty}(\mathfrak{M})=S(\mathfrak{M})$ implies that $\mathfrak{M}$ is $r_{\infty}$-pure where $S(\mathfrak{M})$ is Connes $S$ set [5]. Namely, for any central projection $p$ of $\mathfrak{M}$,

$$
\begin{aligned}
& r_{\infty}(\mathfrak{M} p) \supset r_{\infty}(\mathfrak{M}), \\
& S(\mathfrak{M} p) \subset S(\mathfrak{M}), \\
& r_{\infty}(\mathfrak{M} p) \subset S(\mathfrak{M} p),
\end{aligned}
$$

where the second inclusion is because $\Delta_{\rho}$ for $\mathfrak{M}_{p}$ is always a restriction of $\Delta_{\bar{\rho}}$ for $\mathfrak{M}$ for some $\bar{\rho}$. Hence

$$
r_{\infty}(\mathfrak{M} p)=S(\mathfrak{M} p)=r_{\infty}(\mathfrak{M})
$$

The decomposition $\mathfrak{M}=\Sigma P_{n} \mathfrak{M}$ in Theorem 2 is a partial central decomposition according to asymptotic ratio set into $r_{\infty}$ pure parts [7].

By exactly the same method as the proof of Theorem 2, we can analyze a von Neumann algebra $\mathfrak{M}$ with a cyclic and separating vector $\Omega$, 
such that $\log \Delta$ has exclusively an isolated point spectrum and each $\mathfrak{S}_{a}$ is cyclic for $\mathfrak{M}$. The last assumptions imply that each $\mathfrak{S}_{a}$ is separating for $\mathfrak{M}$ because $J \mathfrak{S}_{a}=\mathfrak{L}_{-a}$ and replaces Step 2 of the proof of Theorem 2. We can proceed up to Step 8 without any further assumption. However Step 10 no longer holds and hence one finds a formula

$$
\left(U^{*} U\right)^{\natural}=e^{-a}\left(A_{a}^{0}\right)^{2}\left(\phi_{a}^{0}\right)^{-1}\left(\left(U U^{*}\right)^{\natural}\right) .
$$

If we make a further assumption that $e^{-a}\left(A_{a}^{0}\right)^{2} \leqq 1$, then we can complete the analysis and we obtain the same conclusion as Theorem 1 except that $\phi_{n}$ no longer satisfies $\phi_{n}(z)=\phi_{n}(1) z$ and $M\left(P_{n} \mathfrak{M}_{0}, \phi_{n}\right)$ corresponds to the case $A_{\phi_{n}}=e^{a_{n} / 2}$ in Theorem 3 .

If $e^{-a}\left(A_{a}^{0}\right)^{2} \leqq 1$ does not hold, we are left with an isomorphism from a subalgebra of $\mathfrak{M}$ onto another subalgebra of $\mathfrak{M}$. It will be of interest to generalize $M(\mathfrak{F}, \phi)$ for such $\phi$.

The construction of $M(\mathfrak{F}, \phi)$ in Section 2 can be generalized to the case where $\mathfrak{M}$ has a commutative semigroup $G$ of injective endomorphisms, which we shall briefly sketch. This situation is relevant to $R_{x} \otimes R_{y} \sim R_{\infty}$ when $\log x / \log y$ is irrational. We assume that

$$
\phi(\mathfrak{F})=\phi(1) \mathfrak{F} \phi(1), \quad \phi \in G
$$

If $\phi_{1}, \phi_{2}$ and $\phi$ are injective endomorphisms of $\mathfrak{M}$, then $\phi \circ \phi_{1}=\phi \circ \phi_{2}$ implies $\phi_{1}=\phi_{2}$. Hence a commutative semigroup $G$ of injective endomorphisms of $\mathfrak{M}$ has an envelopping group $\bar{G}$ such that $\bar{G} \supset G$ and $G$ generates $\bar{G}$. Elements in $\bar{G}$ is a pair $\left(\phi_{a}, \phi_{b}\right)$ of elements $\phi_{a}, \phi_{b} \in G$ with an equivalence relation $\left(\psi \phi_{a}, \psi \phi_{b}\right)=\left(\psi^{\prime} \phi_{a}, \psi^{\prime} \phi_{b}\right)$ for any $\psi, \phi^{\prime} \in G$, where we include an identity mapping 1 of $\mathfrak{F}$ in $G$. The multiplication in $\bar{G}$ is

$$
\left(\phi_{a}, \phi_{b}\right)\left(\psi_{a}, \psi_{b}\right)=\left(\phi_{a} \psi_{a}, \phi_{b} \psi_{b}\right)
$$

and $(\phi, 1)$ is identified with $\phi \in G . \quad 1=(\phi, \phi)$ is the identity in $\bar{G}$.

For each $g \in \bar{G}$, we make a fixed choice $\phi_{a}(g)$ and $\phi_{b}(g)$ such that $g=\left(\phi_{a}(g), \phi_{b}(g)\right)$, where $\phi_{a}(1)=\phi_{b}(1)=1$ and $\phi_{a}\left(g^{-1}\right)=\phi_{b}(g), \phi_{b}\left(g^{-1}\right)=$ $\phi_{a}(g)$ for convenience sake.

The space $\mathfrak{g}$, on which $M(\mathfrak{F}, G)$ is to be defined, is spanned by mutually orthogonal subspaces 


$$
\mathfrak{L}_{g}=p_{g} \Re
$$

where $p_{g}$ is a partially isometric mapping from $\mathfrak{R}$ into $\mathfrak{S}$ such that

$$
E(g) \equiv p_{g}^{*} p_{g}=\phi_{a}(g)(?) \phi_{b}(g)^{\prime}(1),
$$

where $\phi^{\prime}(y)=j_{\Psi} \phi j_{\Psi}(y)$ as before.

In section 2, we have $G=\left\{\phi^{n}, n=0,1, \ldots\right\}, \bar{G}$ is the additive group of integers, $\phi_{a}(n)=\phi^{n}$ for $n \geqq 0, \phi_{a}(n)=1$ for $n \leqq 0, \phi_{b}(n)=1$ for $n \geqq 0$ and $\phi_{b}(n)=\phi^{|n|}$ for $n \leqq 0$.

Faithful representations of $\mathfrak{F}$ and $\mathfrak{F}^{\prime}$ are defined by

$$
\begin{array}{ll}
\pi(x)=\Sigma p_{g} \phi_{a}(g)(x) p_{g}^{*}, & x \in \mathfrak{F}, \\
\pi^{\prime}(y)=\Sigma p_{g} \phi_{b}(g)^{\prime}(y) p_{g}^{*}, & y \in \mathfrak{F}^{\prime} .
\end{array}
$$

$A_{\phi}$ is defined by (2.1) for each $\phi \in G$ and $V$ of Lemma 1 is denoted by $V(\phi)$. It satisfies

$$
\begin{aligned}
& V(\phi)^{*} V(\phi)=1, V(\phi) V(\phi)^{*} \equiv e(\phi)=\phi(1) \phi^{\prime}(1), \\
& V(\phi) Q=\phi(Q) V(\phi), V(\phi)^{*} \phi(Q)=Q V(\phi)^{*}, Q \in \mathfrak{F}, \\
& V(\phi) Q^{\prime}=\phi^{\prime}\left(Q^{\prime}\right) V(\phi), V(\phi)^{*} \phi^{\prime}\left(Q^{\prime}\right)=Q^{\prime} V(\phi)^{*}, Q^{\prime} \in \mathfrak{F}^{\prime}, \\
& {\left[J_{\Psi}, V(\phi)\right]=0,} \\
& V\left(\phi_{1}\right) V\left(\phi_{2}\right)=V\left(\phi_{1} \phi_{2}\right), V(1)=1 .
\end{aligned}
$$

From (6.4) and (6.8), we have

$$
\begin{aligned}
V\left(\phi_{1}\right) V\left(\phi_{2}\right)^{*} & =V\left(\phi_{2}\right)^{*} V\left(\phi_{2}\right) V\left(\phi_{1}\right) V\left(\phi_{2}\right)^{*} \\
& =V\left(\phi_{2}\right)^{*} V\left(\phi_{1}\right) e\left(\phi_{2}\right) .
\end{aligned}
$$

Operators $U(g)$ and $U^{\prime}(g)$ are defined by

$$
\begin{array}{ll}
U(g)=\sum_{g^{\prime}} p_{g g^{\prime}} V\left(\phi_{b}\left(g^{\prime}\right)\right) * V\left(\phi_{b}\left(g g^{\prime}\right)\right) p_{g^{\prime}}^{*}, & g \in \bar{G}, \\
U^{\prime}\left(g^{-1}\right)=\sum_{g^{\prime}} p_{g g^{\prime}} V\left(\phi_{a}\left(g^{\prime}\right)\right)^{*} V\left(\phi_{a}\left(g g^{\prime}\right)\right) p_{g^{\prime}}^{*}, \quad g \in \bar{G} .
\end{array}
$$

They satisfy 


$$
\begin{aligned}
& U(g)^{*}=U\left(g^{-1}\right), U^{\prime}\left(g^{-1}\right)^{*}=U^{\prime}(g), \\
& U(g) U((1, \phi))=U(g(1, \phi)), U(1)=1, \\
& U(g) \pi(x) U((\phi, 1))=U(g(\phi, 1)) \pi(\phi(x)), x \in \mathfrak{F}, \\
& U^{\prime}(g) U^{\prime}((1, \phi))=U^{\prime}(g(1, \phi)), U^{\prime}(1)=1, \\
& U^{\prime}(g) \pi^{\prime}(y) U^{\prime}((\phi, 1))=U^{\prime}(g(\phi, 1)) \pi^{\prime}\left(\phi^{\prime}(y)\right), \quad y \in \mathfrak{F}^{\prime} .
\end{aligned}
$$

Taking adjoint and using (6.12), we also have

$$
\begin{aligned}
& U((\phi, 1)) U(g)=U((\phi, 1) g), \\
& U((1, \phi)) \pi(x) U(g)=\pi(\phi(x)) U((1, \phi) g), \\
& U^{\prime}((\phi, 1)) U^{\prime}(g)=U^{\prime}((\phi, 1) g), \\
& U^{\prime}((1, \phi)) \pi^{\prime}(y) U^{\prime}(g)=\pi^{\prime}\left(\phi^{\prime}(y)\right) U^{\prime}((1, \phi) g) .
\end{aligned}
$$

The von Neumann algebras are defined by

$$
\begin{aligned}
& M(\mathfrak{F}, G)=\{\pi(\mathfrak{F}), U(\bar{G})\}^{\prime \prime}, \\
& M^{\prime}(\mathfrak{F}, G)=\left\{\pi^{\prime}\left(\mathfrak{F}^{\prime}\right), U^{\prime}(\bar{G})\right\}^{\prime \prime}
\end{aligned}
$$

Then

$$
M(\mathfrak{F}, G)^{\prime}=M^{\prime}(\mathfrak{F}, G)
$$

and the vector $\Omega(\mathfrak{F}, G) \equiv p_{1} \Psi$ is cyclic and separating for $M(\mathfrak{F}, G)$ with modular operator and modular conjugation operator given by

$$
\begin{aligned}
& \Delta(\mathfrak{F}, G)=\Sigma p_{g}\left\{\phi_{a}(g) A_{\phi_{a}(g)}\right\}^{2}\left\{j_{\Psi}\left(\phi_{b}(g) A_{\phi_{b}(g)}\right)\right\}^{-2} p_{g}^{*}, \\
& J(\mathfrak{F}, G)=\Sigma p_{g^{-1}} J_{\Psi} p_{g}^{*} .
\end{aligned}
$$

If $A_{\phi}>1$ for all $\phi \in G$, then the set $M(\mathfrak{F}, G)_{0}$ of $Q \in M(\mathfrak{F}, G)$ commuting with $\Delta(\mathfrak{F}, G)$ is $\pi(\mathfrak{F})$ and its center is all $z \in \mathfrak{F}_{c}$ such that $\phi(z)=z \phi(1)$ for all $\phi \in G$.

Sketch of proof We shall present proofs of computations later. $J(\widetilde{F}, G)$ is an antiunitary involution (the choice $\phi_{a}\left(g^{-1}\right)=\phi_{b}(g)$ and $\phi_{b}\left(g^{-1}\right)=\phi_{a}(g)$ 
simplifies the expression of $J$ ) and satisfies

$$
\begin{aligned}
& J(\mathfrak{F}, G) \pi(x) J(\mathfrak{F}, G)=\pi^{\prime}\left(j_{\Psi}(x)\right), x \in \mathfrak{F}, \\
& J(\mathfrak{F}, G) U(g) J(\mathfrak{F}, G)=U^{\prime}(g), g \in \bar{G}, \\
& J(\mathfrak{F}, G) \Delta(\mathfrak{F}, G) J(\mathfrak{F}, G)=\Delta(\mathfrak{F}, G)^{-1} .
\end{aligned}
$$

We have $M^{\prime}(\mathfrak{F}, G) \subset M(\mathfrak{F}, G)^{\prime}$ from

$$
\begin{aligned}
& {\left[\pi(x), \pi^{\prime}(y)\right]=0, x \in \mathfrak{F}, y \in \mathfrak{F}^{\prime},} \\
& {\left[U(g), \pi^{\prime}(y)\right]=0, y \in \mathfrak{F}^{\prime}, g \in \bar{G},} \\
& {\left[\pi(x), U^{\prime}(g)\right]=0, x \in \mathfrak{F}, g \in \bar{G},} \\
& {\left[U\left(g_{1}\right), U^{\prime}\left(g_{2}\right)\right]=0, g_{1} \in \bar{G}, g_{2} \in \bar{G} .}
\end{aligned}
$$

$\Omega(\mathfrak{F}, G)$ is cyclic for $M(\mathfrak{F}, G)$ because

$$
U((\psi, 1)) \pi(\mathfrak{F}) U((1, \phi)) \Omega(\mathfrak{F}, G) \supseteqq p_{(\psi, \phi)} \mathfrak{F} V\left(\phi_{b}\{(\phi, \phi)\}\right) \Psi
$$

is dense in $p_{(\uparrow, \phi)} \Re$. By applying $J(\mathfrak{F}, G)$ and using (6.22) and (6.23), we see that $\Omega(\mathfrak{F}, G)$ is cyclic for $M^{\prime}(\mathfrak{F}, G)$ and hence is separating for $M(\mathfrak{F}, G)$.

By setting $S=J(\mathfrak{F}, G) \Delta(\mathfrak{F}, G)^{1 / 2}$, we have

$$
\begin{aligned}
& S U((\psi, 1)) \pi(x) U((1, \phi)) \Omega(\mathfrak{F}, G) \\
& \quad=U((\phi, 1)) \pi\left(x^{*}\right) U((1, \phi)) \Omega(\mathfrak{F}, G) .
\end{aligned}
$$

In any monomial of $\pi(\mathfrak{F})$ and $U(G)$, we make the following reordering. First factor any $U(g)$ as $U((\psi, \phi))=U((\psi, 1)) U((1, \phi))$ by (6.13). Bring all $U((1, \phi))$ to the right using $(6.14)^{\prime}$ with $g=1$ and $(6.14)^{\prime}$ with $x=1$, where $U((1, \phi) g)$ is again decomposed. Similarly bring all $U((\psi, 1))$ to the left using (6.14) with $g=1$. Collect all $U((1, \phi))$ into one using (6.13) in the form $U\left(\left(1, \phi_{1}\right)\right) U\left(\left(1, \phi_{2}\right)\right)=U\left(\left(1, \phi_{1} \cdot \phi_{2}\right)\right)$. Similarly collect all $U((\Psi .1))$ into one by using $(6.13)^{\prime}$. We then see that $U((\psi, 1)) \pi(x)$ $U((1, \phi))$ are total in $M(\mathfrak{F}, G)$. As we shall show after the following computations, $U((G, 1)) \pi(\mathfrak{F}) U((1, G)) \Omega(\mathfrak{F}, G)$ contains a total set of analytic vectors for $\Delta(\mathfrak{F}, G)^{1 / 2}$. Hence $(6.30)$ shows that $J(\mathfrak{F}, G)$ and $\Delta(\mathfrak{F}, G)$ are 
modular conjugation operator and modular operator for $\Omega(\mathfrak{F}, G)$.

From (6.22), (6.23) and $M^{\prime}(\mathfrak{F}, G) \subset M(\mathfrak{F}, G)^{\prime}$, we have (6.19). The assertion for the case $A_{\phi}>1$ is proved in the same way as the proof of Theorem 3.

Computations. We present proof of those formulas which require more complicated computations.

Formula (6.13). Setting $\phi_{1}=\phi_{b}\left(g^{\prime}\right), \phi_{2}=\phi_{b}\left((1, \phi) g^{\prime}\right), \phi_{3}=\phi_{b}(g(1$, $\left.\phi) g^{\prime}\right)$ and $\psi=\phi_{a}\left((1, \phi) g^{\prime}\right)$, we have

$$
\begin{aligned}
& U(g) U((1, \phi)) \\
& =\sum_{g^{\prime}} p_{g(1, \phi) g^{\prime}} V\left(\phi_{2}\right)^{*} V\left(\phi_{3}\right) \phi_{2}^{\prime}(1) \psi(1) V\left(\phi_{1}\right)^{*} V\left(\phi_{2}\right) p_{g^{\prime}}^{*} \\
& =\sum_{g^{\prime}} p_{g(1, \phi) g^{\prime}} V\left(\phi_{1} \phi_{2}\right)^{*} V\left(\phi_{3}\right) e\left(\phi_{1}\right)\left(\phi_{1} \phi_{2}\right)^{\prime}(1) \phi_{1} \psi(1) V\left(\phi_{2}\right) p_{g^{\prime}}^{*}
\end{aligned}
$$

where we have used (6.1), (6.5), (6.6), (6.9) and (6.8). $e\left(\phi_{1}\right)$ is absorbed into $\left(\phi_{1} \phi_{2}\right)^{\prime}(1)\left(\phi_{1} \phi\right)(1)$ because $\phi_{1}(1) \phi_{1}(\phi(1))=\phi_{1} \phi(1)$ and $\phi_{1}^{\prime}(1) \phi_{1}^{\prime}\left(\phi_{2}^{\prime}(1)\right)$ $=\left(\phi_{1} \phi_{2}\right)^{\prime}(1)$.

There exist $\xi$ and $\eta \in G$ such that

$$
\eta \phi_{a}\left((1, \phi) g^{\prime}\right)=\xi \phi_{a}\left(g^{\prime}\right), \eta \phi_{b}\left((1, \phi) g^{\prime}\right)=\xi \phi_{b}\left(g^{\prime}\right) \phi
$$

Then

$$
\xi \eta \phi_{b}\left((1, \phi) g^{\prime}\right) \phi_{a}\left(g^{\prime}\right)(1)=\xi \eta \phi_{a}\left((1, \phi) g^{\prime}\right) \phi_{b}\left(g^{\prime}\right)(\phi(1))
$$

which implies $\phi_{2} \phi_{a}\left(g^{\prime}\right)(1)=\phi_{1} \phi(\phi(1))$. Since $\phi_{a}\left(g^{\prime}\right)(1) p_{g^{\prime}}^{*}=p_{g^{\prime}}^{*}, V\left(\phi_{2}\right) \phi_{a}$ $\left(g^{\prime}\right)(1)=\phi_{2} \phi_{a}\left(g^{\prime}\right)(1) V\left(\phi_{2}\right)$ and $\phi_{1} \psi(1) \phi_{1} \psi(\phi(1))=\phi_{1} \psi(\phi(1)), \phi_{1}(\psi(1))$ is absorbed into $p_{g^{\prime}}^{*}$ in (6.31). Similarly $p_{g(1, \phi) g^{\prime}} \phi_{3}^{\prime}(1)=p_{g(1, \phi) g^{\prime}}, V\left(\phi_{1} \phi_{2}\right)^{*}$ $V\left(\phi_{3}\right)\left(\phi_{2} \phi_{1}\right)^{\prime}(1)=\phi_{3}^{\prime}(1) V\left(\phi_{1} \phi_{2}\right)^{*} V\left(\phi_{3}\right)$ and hence $\left(\phi_{1} \phi_{2}\right)^{\prime}(1)$ is absorbed into $p_{g(1, \phi) g^{\prime}}$. Using $V\left(\phi_{2}\right) * V\left(\phi_{2}\right)=1$,

$$
U(g) U((1, \phi))=\sum_{g^{\prime}} p_{g(1, \phi) g^{\prime}} V\left(\phi_{1}\right)^{*} V\left(\phi_{3}\right) p_{g^{\prime}}^{*}=U(g(1, \phi))
$$

Formula (6.14). Setting $\phi_{1}=\phi_{b}\left(g^{\prime}\right), \phi_{2}=\phi_{b}\left((\phi, 1) g^{\prime}\right), \phi_{3}=\phi_{b}(g(\phi$, 1) $\left.g^{\prime}\right)$ and $\phi=\phi_{a}\left((\phi, 1) g^{\prime}\right)$, we have 


$$
\begin{aligned}
U(g) & \pi(x) U((\phi, 1)) \\
= & \sum_{g^{\prime}} p_{g(\phi, 1) g^{\prime}} V\left(\phi_{2}\right)^{*} V\left(\phi_{3}\right) \psi(x) \phi_{2}^{\prime}(1) V\left(\phi_{1}\right)^{*} V\left(\phi_{2}\right) p_{g^{\prime}}^{*} \\
= & \sum_{g^{\prime}} p_{g(\phi, 1) g^{\prime}} V\left(\phi_{1} \phi_{2}\right)^{*} V\left(\phi_{3}\right) e\left(\phi_{1}\right) \phi_{1} \psi(x)\left(\phi_{1} \phi_{2}\right)^{\prime}(1) V\left(\phi_{2}\right) p_{g^{\prime}}^{*}
\end{aligned}
$$

As before, $e\left(\phi_{1}\right)$ is absorbed into $\phi_{1} \psi(x)\left(\phi_{1} \phi_{2}\right)^{\prime}(1)$ and $\left(\phi_{1} \phi_{2}\right)^{\prime}(1)$ is then absorbed into $p_{g(\phi, 1) g^{\prime}}$. By an equation similar to (6.32), we have

$$
\phi_{1} \phi(x)=\phi_{2} \phi_{a}\left(g^{\prime}\right) \phi(x)
$$

Hence

$$
\begin{aligned}
U(g) \pi(x) U((\phi, 1)) & =\sum_{g^{\prime}} p_{g(\phi, 1) g^{\prime}} V\left(\phi_{1}\right) * V\left(\phi_{3}\right) \phi_{a}\left(g^{\prime}\right) \phi(x) p_{g^{\prime}}^{*} \\
& =U(g(\phi, 1)) \pi(\phi(x)) .
\end{aligned}
$$

Formula (6.26). Setting $\phi_{1}=\phi_{b}\left(g^{\prime}\right)$ and $\phi_{2}=\phi_{b}\left(g g^{\prime}\right)$, we have

$$
\begin{aligned}
{\left[U(g), \pi^{\prime}(y)\right]=} & \sum_{g^{\prime}} p_{g g^{\prime}}\left[V\left(\phi_{1}\right)^{*} V\left(\phi_{2}\right) E\left(g^{\prime}\right) \phi_{1}^{\prime}(y)\right. \\
& \left.-\phi_{2}^{\prime}(y) E\left(g g^{\prime}\right) V^{*}\left(\phi_{1}\right) V\left(\phi_{2}\right)\right] p_{g^{\prime}}=0
\end{aligned}
$$

where $E\left(g^{\prime}\right)$ and $E\left(g g^{\prime}\right)$ are absorbed into $p_{g^{\prime}}$ and $p_{g g^{\prime}}$ respectively.

Formula (6.28). Setting $\phi_{1}=\phi_{b}\left(g^{\prime}\right), \quad \phi_{1}=\phi_{a}\left(g^{\prime}\right), \quad \phi_{2}=\phi_{b}\left(g_{2}^{-1} g^{\prime}\right)$, $\phi_{2}=\phi_{a}\left(g_{2}^{-1} g^{\prime}\right), \phi_{3}=\phi_{b}\left(g_{1} g^{\prime}\right), \phi_{3}=\phi_{a}\left(g_{1} g^{\prime}\right), \phi_{4}=\phi_{b}\left(g_{1} g_{2}^{-1} g^{\prime}\right)$ and $\psi_{4}=$ $\phi_{a}\left(g_{1} g_{2}^{-1} g^{\prime}\right)$, we have

$$
\begin{aligned}
{\left[U\left(g_{1}\right), U^{\prime}\left(g_{2}\right)\right]=\Sigma p_{g_{1} g_{2}^{-1} g^{\prime}} } & {\left[V\left(\phi_{2}\right)^{*} V\left(\phi_{4}\right) \phi_{2}^{\prime}(1) \phi_{2}(1) V\left(\phi_{1}\right) * V\left(\phi_{2}\right)\right.} \\
& \left.-V\left(\phi_{3}\right) * V\left(\phi_{4}\right) \phi_{3}^{\prime}(1) \phi_{3}(1) V\left(\phi_{1}\right) * V\left(\phi_{3}\right)\right] p_{g^{\prime}}^{*} .
\end{aligned}
$$

Since $\phi_{2}(1) V\left(\psi_{1}\right) * V\left(\phi_{2}\right)=V\left(\psi_{1}\right) * V\left(\psi_{2}\right) \psi_{1}(1)$ and $\phi_{1}(1) p_{g^{\prime}}^{*}=p_{g^{\prime}}^{*}, \psi_{2}(1)$ is absorbed into $p_{g^{\prime}}^{*}$. Likewise, $\phi_{3}^{\prime}(1)$ is absorbed into $p_{g^{\prime}}^{*}, \phi_{2}^{\prime}(1)$ and $\phi_{3}(1)$ are absorbed into $p_{g_{1} g_{2}^{-1} g^{\prime}}$. By (6.9), we have

$$
\begin{aligned}
& {\left[U\left(g_{1}\right), U^{\prime}\left(g_{2}\right)\right]=\Sigma p_{g_{1} g_{2}^{-1} g^{\prime}} } {\left[V\left(\phi_{2} \psi\right)^{*} V\left(\phi_{4}\right) e\left(\phi_{1}\right) V\left(\phi_{2}\right)\right.} \\
&\left.-V\left(\phi_{3} \phi_{1}\right) * V\left(\phi_{4}\right) e\left(\phi_{1}\right) V\left(\phi_{3}\right)\right] p_{g^{\prime}}^{*}
\end{aligned}
$$


Since $\psi_{1}(1) p_{g^{\prime}}^{*}=p_{g^{\prime}}^{*}, \quad V\left(\psi_{2}\right) \psi_{1}(1)=\psi_{2} \psi_{1}(1) V\left(\psi_{2}\right)$ and $\phi_{1}(1) \psi_{1}\left(\psi_{2}(1)\right)$ $=\phi_{1} \phi_{2}(1), \phi_{1}(1)$ in $e\left(\phi_{1}\right)$ is absorbed into $p_{g^{\prime}}^{*}$. Likewise, $\phi_{1}^{\prime}(1)$ in $e\left(\phi_{1}\right)$ is absorbed into $p_{g^{\prime}}^{*}, \psi_{1}^{\prime}(1)$ in $e(\psi)$ and $\phi_{1}(1)$ in $e\left(\phi_{1}\right)$ are absorbed into $p_{g_{1} g_{2}^{-1} g^{\prime}}$. We now have

$$
\left[U\left(g_{1}\right), U^{\prime}\left(g_{2}\right)\right]=\Sigma p_{g_{1} g_{2}^{-1} g^{\prime}}\left[V\left(\phi_{2} \psi_{1}\right)^{*} V\left(\phi_{4} \phi_{2}\right)-V\left(\phi_{3} \phi_{1}\right) * V\left(\psi_{4} \phi_{3}\right)\right] p_{g^{\prime}}^{*}
$$

Let

$$
\begin{aligned}
& \eta_{1} \phi_{2}=\xi_{1} \phi_{b}\left(g_{2}^{-1}\right) \phi_{1}, \eta_{1} \phi_{2}=\xi_{1} \phi_{a}\left(g_{2}^{-1}\right) \psi_{1}, \\
& \eta_{2} \phi_{4}=\xi_{2} \phi_{b}\left(g_{2}^{-1}\right) \phi_{3}, \eta_{2} \psi_{4}=\xi_{2} \phi_{a}\left(g_{2}^{-1}\right) \psi_{3} .
\end{aligned}
$$

Then

$$
\begin{aligned}
\eta_{1} \eta_{2} \psi_{2} \phi_{4} \phi_{1} \phi_{3} & =\xi_{1} \xi_{2} \phi_{a}\left(g_{2}^{-1}\right) \phi_{b}\left(g_{2}^{-1}\right) \phi_{3} \psi_{1} \phi_{1} \phi_{3} \\
& =\eta_{1} \eta_{2} \phi_{2} \phi_{4} \phi_{1} \phi_{3} .
\end{aligned}
$$

Hence $\phi_{2} \phi_{4} \phi_{1} \psi_{3}=\phi_{2} \psi_{4} \psi_{1} \phi_{3}$, which implies

$$
\begin{aligned}
V\left(\phi_{2} \phi_{1}\right) * V\left(\phi_{4} \phi_{2}\right) & =V\left(\phi_{2} \phi_{1} \phi_{1} \phi_{3}\right) * V\left(\phi_{1} \phi_{3} \phi_{4} \phi_{2}\right) \\
& =V\left(\phi_{1} \phi_{3} \phi_{2} \phi_{1}\right) * V\left(\phi_{2} \phi_{1} \phi_{4} \phi_{3}\right) \\
& =V\left(\phi_{1} \phi_{3}\right) * V\left(\phi_{4} \phi_{3}\right) .
\end{aligned}
$$

Hence we have (6.28).

Formula (6.29) Setting $\phi_{1}=\phi_{b}((1, \phi)), \phi_{1}=\phi_{a}((1, \phi)), \phi_{2}=\phi_{b}((\psi, \phi))$ and $\psi_{2}=\phi_{a}((\phi, \phi))$, we have

$$
\begin{aligned}
& U((\psi, 1)) \pi(x) U((1, \phi)) \Omega(\mathfrak{F}, G)= \\
& p_{(\psi, \phi)} V\left(\phi_{1}\right) * V\left(\phi_{2}\right) \phi_{1}^{\prime}(1) \phi_{1}(x) V\left(\phi_{1}\right) \psi
\end{aligned}
$$

where $\phi_{1}(1)$ has already been absorbed into $\phi_{1}(x)$ and $\phi_{1}^{\prime}(1)$ can be absorbed into $p_{(\uparrow, \phi)}$. Since $\phi_{1}=\phi_{1} \phi$ (which follows from (6.32) with $\left.g^{\prime}=1\right)$ we have

$$
\begin{aligned}
V\left(\phi_{1}\right)^{*} V\left(\phi_{2}\right) \psi_{1}(x) V\left(\phi_{1}\right) & =V(\phi)^{*} \phi_{2}(x) V(\phi) V\left(\phi_{2}\right) \\
& =\phi^{-1}\left(\phi(1) \phi_{2}(x) \phi(1)\right) V\left(\phi_{2}\right)
\end{aligned}
$$


where we have used

$$
\begin{aligned}
V(\phi)^{*} x V(\phi) & =V(\phi)^{*} \phi(1) x \phi(1) V(\phi) \\
& =\phi^{-1}(\phi(1) x \phi(1)) V(\phi)^{*} V(\phi)=\phi^{-1}(\phi(1) x \phi(1)) .
\end{aligned}
$$

Let

$$
\eta \phi_{a}((\phi, \phi))=\xi \phi, \eta \phi_{b}((\phi, \phi))=\xi \phi
$$

Then $\xi \eta \psi_{2} \phi=\xi \eta \psi \phi_{2}$ and hence $\psi_{2} \phi=\psi \phi_{2}$. Hence $\phi_{2}(\mathfrak{F}) \supset \psi \phi_{2}(\mathfrak{F})$ implies

$$
\begin{aligned}
& \phi^{-1}\left(\phi(1) \phi_{2}(\mathfrak{\mho}) \phi(1)\right)=\phi^{-1}\left(\phi(1) \phi_{2}(1) \mathfrak{\mho} \phi_{2}(1) \phi(1)\right) \\
& \supset \phi^{-1}\left(\phi(1)\left(\psi \phi_{2}\right)(1) \mathfrak{\mho}\left(\phi \phi_{2}\right)(1) \phi(1)\right)=\psi_{2}(1) \phi^{-1}(\phi(1) \Im \phi(1)) \phi_{2}(1) \\
& =\phi_{2}(1) \mathfrak{F} \phi_{2}(1) .
\end{aligned}
$$

Hence

$$
\begin{aligned}
& U((\phi, 1)) \pi(\mathfrak{\mho}) U((1, \phi)) \Omega(\mathfrak{F}, G) \\
& \supseteqq p_{(\uparrow, \phi)} \phi_{2}(1) \mathfrak{F} \phi_{2}(1) V\left(\phi_{2}\right) \Psi \\
& =p_{(\uparrow, \phi)} \mathfrak{F} V\left(\phi_{2}\right) \Psi .
\end{aligned}
$$

Since $A_{\phi_{2}}$ is positive definite, support of $\phi_{2}\left(A_{\phi_{2}}\right)=\phi_{2}(1)$. Since $J_{\Psi} \phi_{2}(1) \Psi=\phi_{2}(1) \Psi, \mathfrak{F} V\left(\phi_{2}\right) \Psi=\Im \phi_{2}\left(A_{\phi_{2}}\right) \Psi$ is dense in the closure of

$$
\Im \phi_{2}(1) \Psi=\Im j_{\Psi}\left(\phi_{2}(1)\right) \Psi=\phi_{2}^{\prime}(1) \Im \Psi
$$

which is $\phi_{2}^{\prime}(1) \Re$. Therefore (6.29) is dense in $p_{(\psi, \phi)} \Re$.

Formula (6.30) By (6.33) and (6.34), we have

$$
\begin{aligned}
& U((\phi, 1)) \pi(x) U((1, \phi)) \Omega(\widetilde{\mho}, G) \\
& \quad=p_{(\psi, \phi)} \phi^{-1}\left(\phi(1) \phi_{2}(x) \phi(1)\right) \phi_{2}\left(A_{\phi_{2}}\right) \Psi .
\end{aligned}
$$

By multiplying $\Delta(\mathfrak{F}, G)^{1 / 2}$, we obtain

$$
\begin{aligned}
p_{(\phi, \phi)}\left\{\psi_{2}\left(A_{\phi_{2}}\right)\right\} \phi^{-1}\left(\phi(1) \phi_{2}(x) \phi(1)\right) \phi_{2}\left(A_{\phi_{2}}\right) j_{\Psi}\left(\phi_{2}\left(A_{\phi_{2}}\right)\right)^{-1} \Psi \\
=p_{(\psi, \phi)}\left\{\psi_{2}\left(A_{\psi^{\prime} 2}\right)\right\} \phi^{-1}\left(\phi(1) \phi_{2}(x) \phi(1)\right) \Psi
\end{aligned}
$$


Hence

$$
\begin{aligned}
S U((\phi, 1)) & \pi(x) U((1, \phi)) \Omega(\mathfrak{r}, G) \\
= & p_{(\phi, \uparrow)} \phi^{-1}\left(\phi(1) \phi_{2}\left(x^{*}\right) \phi(1)\right) \psi_{2}\left(A_{\psi_{2}}\right) \Psi \\
= & p_{(\phi, \uparrow)} V(\phi)^{*} \phi_{2}\left(x^{*}\right) V\left(\phi \psi_{2}\right) \Psi .
\end{aligned}
$$

Since $\psi_{2} \phi=\psi \phi_{2}$, we have

$$
\begin{aligned}
V(\phi)^{*} \phi_{2}\left(x^{*}\right) V(\phi) & =V\left(\phi \phi_{2}\right)^{*} \phi_{2} \phi_{2}\left(x^{*}\right) V\left(\phi \phi_{2}\right) \\
& =V\left(\phi \phi_{2}\right)^{*} \phi_{2} \phi_{2}\left(x^{*}\right) V\left(\phi \phi_{2}\right) \\
& =V(\phi)^{*} \psi_{2}\left(x^{*}\right) V(\phi) \\
& =V\left(\phi_{b}((1, \phi))\right)^{*} \phi_{2} \phi_{a}((1, \phi))\left(x^{*}\right) V\left(\phi_{b}((1, \phi))\right)
\end{aligned}
$$

where we have used $\phi_{b}((1, \psi))=\phi_{a}((1, \psi)) \psi$ in the last equality.

Hence

$$
\begin{aligned}
& S U((\phi, 1)) \pi(x) U((1, \phi)) \Omega(\widetilde{F}, G) \\
& =p_{(\phi, \uparrow)} V\left(\phi_{b}((1, \psi))\right) * V\left(\phi_{2}\right) \phi_{a}((1, \psi))\left(x^{*}\right) V\left(\phi_{b}((1, \psi))\right) \Psi \\
& =U((\phi, 1)) \pi\left(x^{*}\right) U((1, \phi)) \Omega(\mathfrak{F}, G) \\
& =\{U((\phi, 1)) \pi(x) U((1, \phi))\} * \Omega(\Im, G),
\end{aligned}
$$

where $\phi_{2}=\phi_{a}((\psi, \phi))=\phi_{b}((\phi, \psi))$ is used.

Q.E.D.

We now prove that $U((G, 1)) \pi(\mathfrak{F}) U(1, G)) \Omega(\mathfrak{F}, G)$ contains a total set of analytic vectors for $\Delta(\mathfrak{F}, G)^{1 / 2}$. Let $\phi, \phi \in G, \phi_{2}=\phi_{a}((\phi, \phi)), \phi_{2}=\phi_{b}((\psi$, $\phi))$. Let $E_{\lambda}^{\chi}$ denote the spectral projection of $A_{\chi}, \chi \in G$, It is in the center of $\mathfrak{F}$. Let $p(\lambda)=\phi\left(E_{\lambda}^{\phi_{2}}\right), q(r)=\phi\left(1-E_{r}^{\phi_{2}}\right)$. Then $\lim _{\lambda \rightarrow+\infty} p(\lambda)=1$, $\lim _{r \rightarrow+0} q(r)=1$. Since $\phi_{2} \psi=\phi \psi_{2}$, we have $\phi_{2}(p(\lambda))=\phi\left\{\phi_{2}\left(E_{\lambda}^{\phi_{2}}\right)\right\}$. Hence

$$
\begin{aligned}
& U((\psi, 1)) \pi(p(\lambda) x q(r)) U((1, \phi)) \Omega(\mathfrak{F}, G) \\
& =p_{(\uparrow, \phi)} \psi_{2}\left(E_{\lambda}^{\phi_{2}}\right) \phi^{-1}\left(\phi(1) \phi_{2}(x) \phi(1)\right) \phi_{2}\left(A_{\phi_{2}}\right) \phi_{2}\left(1-E_{r}^{\phi_{2}}\right) \Psi \\
& =p_{(\uparrow, \phi)}\left[\phi_{2}\left(E_{\lambda}^{\phi_{2}}\right) j_{\Psi}\left\{\phi_{2}\left(1-E_{r}^{\phi_{2}}\right)\right\}\right] \phi^{-1}\left(\phi(1) \phi_{2}(x) \phi(1)\right) \phi_{2}\left(A_{\phi_{2}}\right) \Psi,
\end{aligned}
$$


which is obviously an analytic vector for $\Delta(\mathfrak{F}, G)^{1 / 2}$ for $\lambda<+\infty, r>0$. Hence we have a total set of analytic vectors for $\Delta(\mathfrak{F}, G){ }^{1 / 2}$

Group-measure construction. If $G$ is a commutative group of *-automorphisms, then we see that $M(\mathfrak{F}, G)$ defined above is unitarily equivalent to an ordinary group measure construction in the following manner.

$\bar{G}$ is now isomorphic to $G$ with $g \in \bar{G}$ corresponding to $\hat{g} \equiv \phi_{a}(g) \phi_{b}$ $(g)^{-1} \in G$. We have $\phi(1)=\phi^{\prime}(1)=1, p_{g}^{*} p_{g}=1$ and $V(\phi), \phi \in G$ is a unitary representation of $G$. We denote $\bar{V}(g)=V\left(\phi_{a}(g)\right) V\left(\phi_{b}(g)\right) *(=$ $\left.V\left(\phi_{a}(g) \phi_{b}(g)^{-1}\right)\right)$. Since $\phi\left(\mathfrak{F}_{c}\right)=\mathfrak{F}_{c}, \phi\left(A_{\phi}\right)$ is affiliated with $\mathfrak{F}_{c}$.

We define

$$
W=\sum_{g} p_{g} V\left(\phi_{a}(g)\right)^{*} p_{g}^{*}
$$

which is a unitary operator on $\mathfrak{S}$. Then

$$
\begin{aligned}
& W \Omega(\mathfrak{F}, G)=\Omega(\mathfrak{\mho}, G), \\
& W \pi(x) W^{*}=\sum_{g} p_{g} x p_{g}^{*}, x \in \mathfrak{F}^{\prime}, \\
& W \pi^{\prime}(y) W^{*}=\sum_{g} p_{g} g^{-1}(y) p_{g}^{*}, y \in \mathfrak{F}^{\prime}, \\
& W U(g) W^{*}=\sum_{g^{\prime}} p_{g^{\prime} g} \bar{V}(g)^{*} p_{g^{\prime}}^{*}, g \in \bar{G}, \\
& W U^{\prime}\left(g^{-1}\right) W^{*}=\sum_{g^{\prime}} p_{g g^{\prime}} p_{g^{\prime}}^{*}, g \in \bar{G}, \\
& W J(\mathfrak{F}, G) W^{*}=\sum_{g} p_{g-1} \bar{V}(g) J_{\Psi} p_{g}^{*}, \\
& W \Delta(\mathfrak{F}, G) W^{*}=\sum_{g} p_{g} \Delta_{g} p_{g}^{*} .
\end{aligned}
$$

Here $\Delta_{g}=A_{\tilde{g}}^{2}$ and satisfies

$$
\left(\hat{g}\left(\Delta_{g}^{1 / 2}\right) \Psi, \hat{g}\left(x \Delta_{g}^{1 / 2}\right) \Psi\right)=(\Psi, x \Psi), x \in \mathfrak{F}
$$

$\bar{V}(g)$ is defined as a unitary operator satisfying

$$
\bar{V}(g) x \Psi=\hat{g}\left(x \Delta_{g}^{1 / 2}\right) \Psi .
$$

The formulas on the right hand sides of $(6.37) \sim(6.40)$ are usual 
group-measure construction (at least for a commutative $\widetilde{F}$ ) and works even when $\bar{G}$ is non-commutative. Formulas (6.41) and (6.42) give modular conjugation and modular operators also for non-commutative $\bar{G}$.

Example. Let $R_{x}=\bigotimes_{g \in G}\left(R_{g}, \Omega_{g}\right)$ where $G$ is a countable set for the moment, $R_{g}$ is a type $I_{2}$ factor and $\Omega_{g}$ has a spectrum $\lambda$ and $(1-\lambda)$ relative to $R_{g}$ (independent of $g$ ) where $\lambda(1-\lambda)^{-1}=x$. Since the modular operator for $\Omega=\otimes \Omega_{g}$ has a spectrum at $S_{x}=\{0\} \cup\left\{x^{n} ; n=0, \pm 1, \ldots\right\}$ and $R$ is asymptotically abelian relative to any one parameter non-compact shift of $G$, the condition $3=Z_{0}$ is satisfied by Theorem 2 and $\left(R_{x}, \Omega\right) \sim(M(\mathfrak{F}, \phi)$, $\Omega(\mathfrak{F}, \phi)$ ) for a hyperfinite finite factor $\mathfrak{F}$ and its endomorphism $\phi$. The hyperfiniteness of $\mathfrak{F}$ is easily seen by expressing it by a group measure construction where the group is generated by an ascending sequence of finite groups.

Now let $G$ be a group and $V(g), g \in G$ is a unitary operator on $\underset{g^{\prime}}{\otimes}\left(\mathfrak{g}_{g^{\prime}}, \Omega_{g^{\prime}}\right)$ which shifts indices $g^{\prime} \in G$ by left multiplication, namely

$$
V(g) \pi_{g_{1}}\left(Q_{1}\right) \ldots \pi_{g_{n}}\left(Q_{n}\right) \Omega=\pi_{g g_{1}}\left(Q_{1}\right) \ldots \pi_{g g_{n}}\left(Q_{n}\right) \Omega
$$

where all $R_{g}, \Omega_{g}$ are identified with a single $I_{2}$ factor $R_{0}$ and a vector $\Omega_{0}, \pi_{g_{k}}$ is a natural representation of $R \sim R_{g_{k}}$ on $\otimes\left(\mathfrak{g}_{g}, \Omega_{g}\right)$ and $Q_{k} \in R_{0}$. Then $V(g) \Omega=\Omega, V(g) R_{x} V(g)^{*}=R_{x}$ and hence $V(g)\left(R_{x}\right)_{0} V(g)^{*}=\left(R_{x}\right)_{0}$ because $V(g)$ commutes with modular operator $\Delta$ for $\Omega$. It also commutes with the modular conjugation $J$ for $\Omega$.

Consider $M\left(R_{x}, G\right)$ constructed in exactly the same way as $M(\mathfrak{F}, G)$ by $(6.36) \sim(6.40)$ and $M(\mathfrak{F}, G)=(\pi(\mathfrak{F}), U(G))^{\prime \prime}$. Then (6.41) and (6.42) give modular conjugation operator and modular operator for $\Omega\left(R_{x}, G\right)$ where $J_{\Psi}$ and $\Delta_{g}$ are to be replaced by $J$ and $\Delta$. In particular $\Delta\left(R_{x}, G\right)$ has a spectrum $S_{x}$.

The set $M\left(R_{x}, G\right)_{0}$ of modular invariant elements of $M\left(R_{x}, G\right)$ is $M\left(\left(R_{x}\right)_{0}, G\right)$. An isometric operator $U \in R_{x}$ inducing an injective endomorphism $\phi$ such that $\left(R_{x}, \Omega\right) \sim\left(M\left(\left(R_{x}\right)_{0}, \phi\right), \Omega\left(\left(R_{x}\right)_{0}, \phi\right)\right)$ also induces an injective endomorphism of $M\left(R_{x}, G\right)_{0}$ by

$$
\pi(U)^{*} Q \pi(U)=\bar{\phi}(Q), Q \in M\left(R_{x}, G\right)_{0}
$$

The pair, $M\left(R_{x}, G\right)$ and $\Omega\left(R_{x}, G\right)$, is then unitarily equivalent to the pair, 
$M(\overline{\mathfrak{F}}, \bar{\phi})$ with $\overline{\mathfrak{F}} \equiv M\left(\left(R_{x}\right), G\right)_{0} \sim M\left(\left(R_{x}\right)_{0}, G\right)$ and $\Omega(\overline{\mathfrak{F}}, \bar{\phi})$. The case where $G$ is a free group of two generators is given by Pukanszky. Since $M\left(R_{x}, G\right)$ for this case does not have property $L$ its asymptotic ratio set is $\{0\}$.

\section{Acknowledgement}

The author would like to thank members of Department of Mathematics, Queen's University, where this work has been done, for helpful discussions and warm hospitality. The author is indebted to Dr. Nielsen for pointing out a connection with group measure construction and with Pukanszky's example.

The author learned after completion of this work that Takesaki [8] has investigated $M(\mathfrak{F}, \phi)$.

\section{References}

[1] Araki, H., Publ. RIMS Kyoto Univ. 6 (1970/71), 443-460.

[2] Araki, H., Rubl. RIMS Kyoto Univ. 8 (1972/73), 439-469.

[3] Araki, H., Commun. math. Phys. 28 (1972), 267-278.

[4] Araki, H. and E. J. Woods, Publ. RIMS Kyoto Univ. 4 (1968), 51-130.

[5] Connes, A., C.R. Acad. Sc. Paris 273 (1971), 900-903.

[6] Connes, A., C. R. Acad. Sc. Paris 274 (1972), 175-177.

[7] Nielsen, O. A., Can. J. Math. 23 (1971), 598-607.

[8] Takesaki, M., The structure of a von Neumann algebra with a homogeneous periodic state. (Preprint.) 